\title{
Cross-layer design for reducing delay and maximizing lifetime in industrial wireless sensor networks
}

\author{
Jiawei Tan ${ }^{1}$, Anfeng Liu ${ }^{*}$ (D), Ming Zhao ${ }^{2}$, Hailan Shen ${ }^{1}$ and Ming $\mathrm{Ma}^{3}$
}

\begin{abstract}
Low delay and long lifetime are a very important issue for industrial wireless sensor networks (IWSNS) in which it require long-time monitoring of industrial sites and respond quickly to events that is monitored; therefore, high delay communications can cause serious damage to property and personnel at industrial field. Due to delay, lifetime, and other performance involved to multiple layers, it is difficult to optimize from a single layer. Therefore, a cross-layer design optimal scheme for reducing delay and maximizing lifetime (RDML) scheme is proposed for IWSNs which is from several layers such as transmitted power, duty cycle, and node deployment positions to optimize the network performance of delay and lifetime etc. Firstly, due to the node which sends a packet within a cycle, different duty cycle leads to different selection of the modulation level, resulting in different power consumption efficiency of transmitting data. Through careful analysis, the optimal value of the duty cycle is given which has the lowest energy consumption per bit. In fact, the energy consumption of the node is not balanced. Therefore, an optimization method of changing the duty cycle is proposed. In this paper, larger duty cycle is chosen for nodes with residual energy to improve the reliability of data transmission, reducing the probability of data retransmission, so that the network delay can be reduced in IWSNs. Third, based on the previous analysis, a network optimization deployment algorithm is proposed, which not only maximizes the energy efficiency of a single node but also maximizes the network lifetime and the total network energy efficiency. Both our comprehensive theoretical analysis results indicate that the performance of RDML scheme is better than the previous studies. Relative to equal distance and optimal duty cycle scheme, the RDML scheme can reduce the delay by $19-30 \%$ and increase the lifetime by more than $43 \%$.
\end{abstract}

Keywords: Industrial wireless sensor networks, Duty cycle optimal, Network performance optimization, Transmitted power, Low delay, Lifetime

\section{Introduction}

Industrial wireless sensor networks (IWSNs) are important components of Internet of things (IoT) [1-9] which leverage the ubiquity of sensor-equipped devices to collect information at low cost and provide a new paradigm for solving the complex sensing applications from the significant demands of industrial applications such as $[2,4]$ surveillance systems [10-15], intelligent traffic management $[16,17]$, and automated vehicles in environmental transportation [18-20]. The wireless sensor networks have been developed for a long time [21-26]; with the

\footnotetext{
* Correspondence: afengliu@mail.csu.edu.cn

'School of Information Science and Engineering, Central South University, Changsha 410083, China

Full list of author information is available at the end of the article
}

development of wireless portable devices and sensor technology, the application of wireless sensor network in the industrial field becomes the focus of attention $[2,4]$. Industrial wireless sensor networks (IWSNs) is emerging in this background; it does not require wiring to be deployed at any time and has simple requirements for complex industrial sites; the device is small and easy to deploy and has powerful functions that can be used to detect and monitor a variety of visible and invisible physical phenomena in close range and high precision; and the strong practicality makes it to have broad application prospects in various fields of industrial production $[1,2,9$, 10], especially with the rise of cloud computing [27] and fog computing [28-30], to make its development face greater opportunities. 
Industrial wireless sensor networks also face similar problems with wireless sensor networks, and because of the different application situations, there are some differences between IWSNs and WSNs [2]. Firstly, high energy efficiency is urgently required for IWSNs [2]. In IWSNs, sensors are powered by battery; therefore, the energy is limited [31, 32]. But what is more troublesome is that the sensors are placed in a variety of industrial equipment or production workshop. These industrial equipment and workshops were not designed to provide space for these sensors; thus, these sensing nodes can only be required to adapt to the industrial production environment. Therefore, it is required to be smaller in size than other application to adapt to industrial production. But reducing the size of the sensors means that the battery size also decreases [31], and the battery size determines the energy stored in the battery; therefore, in IWSNs, it is very important to design a more efficient energy system [31-33]. The same as wireless sensor networks, the energy consumed by data communications is the most of the energy consumed by the entire system $[34,35]$. Thus, the key to reduce energy consumption and increasing network lifetime is how to maintain efficient communications [36-40, 42]. In IWSNs, sensor nodes can send perceived data to a hub in a one-hop method and can also use multi-hop routing to the control center (CC) [36-39, 41, 42]. Therefore, how to reduce the energy consumption of routing is the key to improving lifetime [43-45].

IWSNs not only require high energy efficiency but also have special needs for network performance [46]. As IWSNs are mainly used in industrial production monitoring and control process, there are many industrial processes in high-temperature and high-pressure environment, and the control process requires extremely sensitive monitoring physical phenomena, and sending out control information requires that time interval is less than industrial milliseconds, that is, the perception of information to the control center and the feedback control information to the control equipment delay is very strict. Because of the large delay which may lead to serious disasters $[8,11,18,24,36,47]$, such as monitoring of industrial boiler temperature and industrial furnace metallurgy, if the control is not timely, may lead to explosion of the boiler, the product quality does not meet the requirements and results in waste. It affects production schedule, wastes production material, destroys industrial production equipment, and seriously causes death to people. Thus, in addition to energy efficiency $[9,22,36,37]$, in IWSNs, the performance metrics such as lifetime and delay are referred to as quality of services (QoS) requirements which is actually important for IWSNs [44, 46, 48].

There are already some studies on energy-efficient QoS for IWSNs [49-51], such as energy-efficient QoS awares routing and delays reduction algorithm [52]. However, there are several key issues in these studies that deserve further study. (1) Most studies are optimized from a layer, so there is a limitation for optimal performance. There are many studies on the performance optimization of IWSNs and wireless sensor networks. Existing are for the optimization of the MAC layer, such as research on the optimization of duty cycle of nodes [53], research on the competition window, and research on the adjustment of slot frame structure for MAC. In optimizing the MAC layer, the main goal is to reduce energy consumption and reduce delay and conflict rates. Existing are for the optimization of the network layer, such as selecting the appropriate relay nodes to make the energy consumption balanced. However, there are few studies that optimize IWSNs from multiple layers. Obviously, if IWSNs can be optimized from multiple layers, the QoS optimization will be better [49-51]. (2) Although reducing the energy consumption of nodes is an optimization goal, this is not the ultimate goal. The ultimate goal of energy optimization is to increase network lifetime rather than to reduce energy consumption. Although many methods are proposed to reduce energy consumption [9, 22, 36, 37], few studies have been optimized from the overall view of the network. From the overall network, in general, the node near the control center is needed to forward data that is from the node away from the control center, so its energy consumption is greater than that of the other nodes. Therefore, although the node near the control center has low energy consumption, the node away from the control center has residual energy. Thus, in this case, the transmit power of the node far from the control center is increased, so that the bit error rate of the node transmission is reduced and the data retransmission times are reduced [49, 51]. So, the data transmission delay is reduced, and the overall network performance is optimized. (3) Although there are many researches on the optimization of network performance from node deployment, most of them have not been optimized from MAC layer [54]. This paper further studies the deficiencies of the previous studies, and the main innovation of the paper is as follows:

(1) Firstly, the optimal duty cycle of the nodes is obtained from the theoretical analysis when the energy consumption of per bit data successfully received is smallest. Because the data transmitted by a node in a cycle is constant, so the duty cycle is larger and the required modulation level is lower; conversely, the smaller the duty cycle, the higher the modulation level required. And the duty cycle and modulation level is related to energy consumption. Therefore, in this paper, the optimized duty cycle is obtained theoretically which can make the lowest energy consumption per bit transmission. 
(2) Secondly, a cross-layer design optimal scheme is proposed to optimize the delay of network and energy efficiency for IWSNs. RDML scheme first selects the optimized duty cycle for each node to maximize the energy efficiency of the data transmission. Then, according to nodes in the network at different locations, the energy consumption of nodes is unbalanced. Greater duty cycle for nodes with residual energy is needed to improve data transfer reliability and reduce delay. Because the node has residual energy, the duty cycle is not to minimize the energy consumption of the sending unit bit at this time. Therefore, increasing the node duty cycle, although more energy is consumed and no effect on network lifetime, can effectively reduce the number of data retransmissions, which can effectively reduce the delay.

(3) Thirdly, a strategy of network deployment is proposed. By unequal deployed nodes, the energy consumption of the network is optimized.

(4) Through our extensive simulation study, the delay and energy efficiency can be enhanced simultaneously using the proposed RDML scheme. RDML scheme selects the optimized parameters for multiple layers and takes full advantage of the residual energy to improve network performance. Comparing with the previous method, the energy utilization rate has reached more than $43 \%$. The delay can be reduced by $19-30 \%$. More importantly, the performance is enhanced without reducing the network lifetime, which is difficult to be achieved in the previous studies.

The rest of this paper is organized as follows: a simple description of the method is presented in Section 2. In Section 3, the related works are reviewed. The system model and problem statement are described in Section 4. In Section 5, the RDML scheme is presented to crosslayer optimization. The performance analysis of RDML scheme is provided in Section 6. We present our conclusions in Section 7.

\section{Methods}

In IWSNs, the energy consumption of sensor nodes is unbalanced, causing the low energy efficiency, and it requires lower delay. Therefore, a method of reducing delay and maximizing network lifetime is proposed. In a typical network, the nodes near sink forward more data packets, so these nodes will consume more energy and thus determine the network lifetime. Therefore, reducing the distance of these nodes to the sink node reduces the energy consumption of these nodes and improves the network lifetime. However, it is difficult to balance the energy consumption of each node through node deployment, so there will still be some nodes that have residual energy; therefore, by mathematically analyzing, adjusting the duty cycle of node can affect the energy consumption and also affect the delay. In the previous strategy, the duty cycle of nodes is of optimal value, so increasing the duty cycle of nodes can increase the energy consumption and will reduce the delay. So, increasing the duty cycle of nodes that have residual energy cannot reduce the network lifetime and can reduce the delay. The background and related work on this method can be found in Section 3 .

\section{Background and related work}

The background work is closely related to Ref. [53]. In these sensor networks, the sensor nodes use periodic sleep/ awake operations to save energy. Considering the periodic time used by the node is presented by $T$, using $T_{\text {on_time }}$ to represent the time of node in active mode, the duty cycle of node is $\tau=T_{\text {on time }} / T$. When the node is in sleep mode, its energy consumption is minimal and can be ignored. When the node is in active mode, the energy consumption is large, especially when receiving data and sending data. However, the node cannot communicate in sleep mode. The node can receive data and send data only in active mode. The same as in Ref. [53], the node can forward a packet in a cycle. Due to the different bits of the data packet, the node adopts the method of changing the modulation level to send a packet in a cycle. Specifically, it is assumed the node unit time to send is $T_{s}$ and the channel bandwidth $B$ equals $1 / T_{s}$, because the time of the node work in a cycle is $T_{\text {on }}$ time. In order to ensure that in the time of $T_{\text {on time }}$ to send a length of $L$ data packets, it must make the number of bit per symbol $b=L /\left(B T_{\text {on_time }}\right)$. Therefore, sending a packet of length $L$ in a cycle is guaranteed.

In fact, this model has been applied in many applications $[49,55]$. The model used by Ruifeng Zhang et al. [55] is similar to this model. One of the main research topics in Ref. [55] is the influence of node transmit power on energy consumption and the reliability of data transfer. The transmit power of the node mainly affects the signal strength of the receiving node, that is, the signal-to-noise ratio of the received signal. In general, the greater the signal-to-noise ratio of the received node, the higher the reliability of the receiving data. However, to increase the signal-to-noise ratio of the receiver node, it is necessary to increase the transmission power of the sending node, and increasing the node's transmit power will increase the energy consumption. When the receiver signal-to-noise ratio is relatively small, the rate of success in receiving data is very low and the rate of the receiver's success in receiving data will increase a lot while the transmit power increases a little. When the signal-to-noise ratio is larger and the rate of success receiving data is larger, even if the sender transmit power is greatly increased, the reception success 
rate of the receiver also increased little. Therefore, Ruifeng Zhang et al. [55] gives the optimization result of how to make the energy consumption of the unit bit to be transmitted successfully.

There are some similar studies, for example, Chen et al. [56] which studied in a linear network, on how to choose the optimal distance that makes the total energy consumption in the multi-hop network minimum. Obviously, this work is similar to the previous research work; in a linear network, the method of minimizing the energy consumption of a single node can make the total energy consumption of the network minimum, but not necessarily maximize the network lifetime. Simultaneously, the optimal transmission range of a node is affected by multiple channel parameters; thus, Ref. [57] also shows how to choose optimized channel parameters to reduce the energy consumption. Ref. [58] defines a method known as a Bit-Meter-per-Joule metric to evaluate energy efficiency. The authors found the network topology and the nodal density, and the transceiver characteristics affect energy consumption; therefore, optimizing energy consumption needs to be optimized from multiple aspects. Xue Chen et al. [58] consider that minimizing the node energy consumption does not necessarily enable the network to achieve high performance and to maximize the network lifetime is the goal of optimization. This is similar to Yunxia Chen et al. [58] which proposed utilization efficiency which is defined as network lifetime per unit deployment cost. Therefore, the difference between their strategy and previous researches is not to reduce the energy consumption of nodes but to balance the energy consumption of nodes and maximize the network lifetime. Because there is a phenomenon in the sensor network that seriously damages the network lifetime which is called "energy hole," that is, the energy consumption of nodes in the near-sink area is much higher than that in the other areas, these nodes die early and make the entire network prematurely die. And on the other hand, nodes in the far-sink area have a lot of residual energy; therefore, Ref. $[49,50]$ proposes two approaches to optimize network performance for this situation. The first is a cross-layer optimization method proposed to minimize the energy consumption of transmitting a bit data by selecting the optimal transmit power. Then, selecting the high transmit power for nodes with energy remaining in the far-sink area can effectively improve the data transmission reliability. This strategy improves the reliability of data transmission while maintaining high network lifetime. In addition, the reliability of data transmission is also related to the data transmission distance. If the transmit power of the sender is increased, when the distance between the nodes is far, better transmission efficiency can be maintained but this time will consume more energy. Thus, Ref. [49, 59] also proposed a method to optimize the network performance from the node deployment. In their approach, for nodes near-sink, there is a smaller distance between the nodes and a larger distance between the nodes far-sink. In this case, energy consumption per bit data transmission in nearsink node is small, while nodes in far-sink area have energy surplus. Therefore, using larger transmit power and larger distance between nodes can still maintain a higher network lifetime and data transmission reliability. The lifetime is improved and data transmission reliability is guaranteed from overall network; the similar study can also be found in Ref. [50]. This paper discusses the cross-layer optimization strategies for energy-efficient and reliable data transmission in AWGN (additive white Gaussian noise channels), RBF (Rayleigh block-fading channels), and RFF (Rayleigh fast fading channels).

Another important issue associated with this paper is the delay $[8,11,18,24,36,60]$. Reducing the delay has been one of the focuses of IWSN research. There are many factors that affect delay. This paper summarizes the following factors:

(1) First, the reliability of data transmission is one of the important factors that affects delay. The main reason that transmission reliability affects data transmission is that if there is a high reliability of data transmission, the data need to be retransmitted less, and multiple retransmissions will lead to the delay of linear growth. Therefore, an important way to reduce the delay is to improve the reliability of the network transmission. The reliability of network transmission is also related to many factors in the network. It is related to the transmit power of nodes. When the transmit power of nodes is large, the signal-to-noise ratio of the wireless channel is large and the signal of the receiver to receive data is strong. Therefore, if the bit error rate of data transmission is low, this will effectively reduce the delay but at this time need to consume more energy, so it can be seen the energy consumption is related to the delay of network.

(2) Second, the method of node retransmission also affects delay. If the node transmit power is fixed, the data transmission reliability is fixed. At this time, some mechanism needs to be taken to ensure the data transmission meets the requirements of the application. The most commonly used measure are the following categories: (a) Retransmission mechanism [34]. The retransmission mechanism is one of the most important ways to guarantee the reliability of the network transmission. The principle of retransmission mechanism is once the data packet is sent, the sender uses some mechanism to 
determine whether the packet is successfully received by the receiver and, if successfully received by the receiver, send the next packet, otherwise retransmitting the current packet. In this method, the receiver sends an ACK to the sender after receiving the packet, which presents data packet was successfully received. The sender does not receive the expected ACK within a fixed time then resends the data packet. The above process continues until the receiver has successfully received the data packet or the number of retransmissions exceeds the maximum predetermined number of retransmissions [34]. The disadvantage of this method is sending of one data packet ends with the ACK signal received by the sender or the number of retransmissions exceeds the set threshold to abandon the sending of this packet. The waiting time between each transmission needs to be more than round-trip time (RTT). Thus, in a link with high packet error rates, multiple retransmission causing its network delay is large [34]. There are many more methods to improve this approach as in Ref. [30]. (b) Network coding techniques are an effective and reliable guarantee mechanism based on redundant coding [34]. In network coding techniques, the source node encodes the packets with some redundant level. The destination node decodes the packets to retrieve the original packets. Because the data sent by the source node is redundant, thus, even if some data is lost in the lossy nature of wireless channels, it will not affect the destination node correctly receiving the data. Network coding techniques have the advantage that the source node can send the data at once and the destination node can be received with high reliability and so, compared with retransmission mechanism, can reduce the delay. But the cost is that the nodes need to encode and decode and the data that is transmitted is redundant, resulting in higher computation and energy consumption for nodes. (c) Multiple transmission mechanism. This mechanism is generally used less and mainly used to protect the safety of data transmission. In this method, the data packets are sent through multiple paths at the same time. As long as any one path successfully reaches the destination, the data transmission is successful [61]. The advantage of this approach is that the delay is small, whereas the disadvantage is that the energy consumption of the network is high.

(3) The third is the adjusting of the node duty cycle. The duty cycle has an important influence in the network $[24,53]$. The effect of duty cycle on delay is mainly reflected in the following aspects. Because the node adopts sleep/active mode to work and the node in the sleep state cannot send and receive data, therefore, when the data of the sender needs to be transmitted, if the receiver is in the sleep state, the sender needs to wait for its wake to be transmitted, thus increasing the delay. Therefore, to reduce the delay, we need to increase the duty cycle. But increasing the duty cycle will consume more energy of the nodes. Thus, there is also an optimization problem between the duty cycle and the energy consumption of nodes; such studies can be found in the literature $[24,53]$.

From the above, the network performance optimization involves all aspects of the network. Energy consumption is an important aspect of network performance. The difference between this study and the previous studies are the following:

(1) Most of the above studies mainly optimize the energy consumption of nodes by optimizing the energy consumption per bit. However, reducing the energy consumption of a single node does not necessarily result in optimal network performance. Although some studies have proposed to optimize the network performance as a whole, their research mainly focuses on the transmission power of the nodes without optimizing from the modulation level. At the same time, there is a close correlation between the modulation level and the duty cycle of the node. Therefore, a new method of overall performance optimization from the duty cycle is proposed.

(2) In addition to optimizing the network parameters, a comprehensive optimization method from the node deployment is also presented. And applying our method to different uncoded modulation systems, i.e., MQAM (M-ary quadrature amplitude modulation) and MPSK (M-ary phase shift keying), confirmed its effectiveness.

\section{The system model and problem statement}

\subsection{The energy consumption model}

The energy consumption model in this paper is the same with Ref. [53]. According to Ref. [53], The sensor node transmission period $T$ consists of three parts which are the time of transient mode $T_{\text {start }}$, transmit mode $T_{\text {on_time, }}$ and standby mode $T_{\text {stby }}$.

$$
T=T_{\text {start }}+T_{\text {on_time }}+T_{\text {stby }}
$$

Power consumption associated to the described modes are denoted as $P_{\text {start }}, P_{\text {on_time, }}$ and $P_{\text {stby. }}$. In general, researchers assumed the standby mode is null for simplification [53]. Therefore, the equation for the energy consumed is: 


$$
\begin{aligned}
E_{\text {total }} & =P_{\text {on_time }} T_{\text {on_time }}+P_{\text {start }} T_{\text {start }} \\
& =\left(P_{\text {Tx }}+P_{\text {circuit }}+P_{P A}\right) T_{\text {on_time }} \\
& +P_{\text {circuit }} T_{\text {start }}
\end{aligned}
$$

where

$P_{\mathrm{Tx}}$ is the power of data transmission. $P_{\text {circuit }}$ is the circuit power for transmitter and receiver block without considering the amplifier. Because the $T_{\text {start }}$ is very small, it only needs to compute the frequency synthesizer with the higher power in the circuit.

$P_{P A}$ is the power of amplifier. The amplifier power is given by:

$$
P_{\mathrm{PA}}=\beta P_{\mathrm{Tx}}=\left(\frac{\xi}{\eta}-1\right) P_{\mathrm{Tx}}
$$

where $\eta$ represents the drain efficiency of the amplifier and $\xi$ is the peak to average ratio which is expressed as a function of constellation size $M$ as $\xi=3\left(\frac{\sqrt{M}-1}{\sqrt{M}+1}\right)$ for MQAM and MPSK modulation technique [53].

Therefore, the energy consumption for a transmission period $T$ in MQAM/MPSK modulation is

$$
\begin{aligned}
E_{\text {total }} & =(1+\beta) P_{\text {Tx }} T_{\text {on_time }}+P_{\text {circuit }} T_{\text {on_time }} \\
& +2 P_{\text {syn }} T_{\text {start }}
\end{aligned}
$$

where $T_{\text {on_time }}$ can be obtained from the duty cycle $\tau$

$$
\tau=\frac{T_{\text {on_time }}}{T}
$$

The power of the signal in the output of the transmitter is calculated by the equation of $k$ th path loss model [53].

$$
P_{\mathrm{Tx}}=P_{\mathrm{rx}} G_{d}
$$

$G_{d}=G_{1} d^{k} M_{1}$, where $d$ is the distance between two communicating nodes, the exponent order $k$ is between 2 and $4, G_{1}$ is the gain factor, and $M_{1}$ is the link margin.

The relationship between the received power and the signal-to-noise ratio is as follows:

$$
S N R=P_{\mathrm{rx}} /\left(2 B N_{f} \sigma^{2}\right)
$$

Therefore, the received power can be obtained from signal-to-noise ratio, and according to reference [53], the bit error probability evaluated in the case of AWGN channel is expressed in terms of average value of the transmitted energy as:

\section{(1)For M-ary QAM}

$$
P_{e} \approx \frac{4}{b}\left(1-\frac{1}{\sqrt{M}}\right) e^{\left(-\frac{3}{M-1}\right) \frac{\mathrm{SNR}}{2}}
$$

(2)For M-ary PSK

$$
P_{e} \approx \operatorname{erfc}\left(\sqrt{\mathrm{SNR}} \sin \left(\frac{\pi}{2 M}\right)\right)
$$

where the number of bit per symbol $b=\frac{L}{B \tau T}$ and the constellation size $M=2^{b}$.

Thus, the signal-to-noise ratio can be obtained from the duty cycle. Then, according to Eqs. (6) and (7), the relationship between the duty cycle and the transmitter power can be obtained. So through the duty cycle, get the total energy consumption $E_{\text {total }}$.

\subsection{Realistic unreliable link model}

The unreliable radio link probability $(p l)$ is defined using the packet error rate (PER) [55].

$$
p l\left(\gamma_{x, x^{\prime}}\right)=1-\operatorname{PER}\left(\gamma_{x, x^{\prime}}\right)
$$

where $\gamma$ is the signal-to-noise

$$
p l(\gamma)=\left(1-P_{e}\right)^{L}
$$

According to Ref. [53], the delay between two communicating nodes is:

$$
\varpi=1 / p l(\gamma)
$$

The unreliable link models are approximated for AWGN channels. Therefore, the BER (bit error rate) under MQAM modulation technique or MPSK modulation technique can be obtained from Eq. (8) or (9).

\subsection{Problem statement}

According to Ref. [53], the network lifetime is related to the node which has the maximum energy consumption in the network. When the energy consumption of all nodes is balanced, the network lifetime can achieve an optimization value.

(1) The first goal of this paper is to maximize the network lifetime. The network lifetime is defined as $l$ and the energy consumption of sensor nodes as $E_{i}$. It is clear to maximize the network lifetime is to minimize the energy consumption of the node with the largest energy consumption, which can be expressed as $\min \left\{\max \left(E_{i}\right)\right\}$. When the energy consumption of the nodes is balanced, the system performance is maximized. Therefore, the goal of this paper is to make the energy consumption of the nodes equal, which is $E_{i}=E_{j} \mid \forall i \neq j, i, j \in$ $N(N=\{1,2, \ldots m\}$ consists of all nodes in the network). At the same time, the network lifetime is maximized by adjusting the node duty cycle $\tau$, that 
is, choosing the optimized duty cycle maximizes the network lifetime.

In summary, our first goal is shown in Formula (12).

$$
\left\{\begin{array}{c}
\max (l)=\min \left\{\max \left(E_{i}(\tau)\right)\right\} \mid i \in N \\
\text { s.t. } \\
E_{i}=E_{j} \mid \forall i \neq j, i, j \in N
\end{array}\right.
$$

(2) The equidistant deployment of the network, due to each node in network forwarding different amount of data, will result in each node having different energy consumption. However, the network lifetime only related to the largest energy consumption node. Therefore, a strategy is considered in which the duty cycle of nodes in a network is different, $\exists \tau_{i} \neq \tau_{j} \mid \forall i \neq j, i, j \in N$. We define $\varpi\left(\tau_{i}\right)$ as the delay of node $i$. Then, Eqs. (8) and (9) combined with Eqs. (10) and (11) can deduce the delay. So, our second goal is as much as possible to improve the duty cycle $\tau_{i}$ of each node without affecting the network lifetime, thus reducing the network delay $\delta$.

As followed from the above, our second goal is shown in Formula (13).

$$
\left\{\begin{array}{c}
\min (\delta)=\min \left(\sum \varpi\left(\tau_{i}\right)\right) \mid i \in N \\
\text { s.t. } \quad E_{i}\left(\tau_{i}\right) \leq \max \left(E_{j}\left(\tau_{\mathrm{opt}}\right)\right) \mid i, j \in N
\end{array}\right.
$$

\section{The design of the RDML scheme}

\subsection{The RDML scheme for linear network}

The first network studied in this paper is the linear network. The linear network topology is shown in Fig. 1. There are $n+1$ nodes, that is, sink, $S_{1}, S_{2} \cdots S_{n}$, and $d_{i}$ is the transmission distance between $S_{i}$ and $S_{i-1}$.

Each node except sink in the linear network generates a data packet in a data collection round and transmits it to sink. Therefore, the more the node is close to the sink, the more data is forwarded. Therefore, if the parameter for each node is the same, the node which is nearer the sink consumes more energy (Table 1).

For MQAM, according to Eqs. (4)-(8), the energy consumed to transmit a packet when using MQAM technology is as:

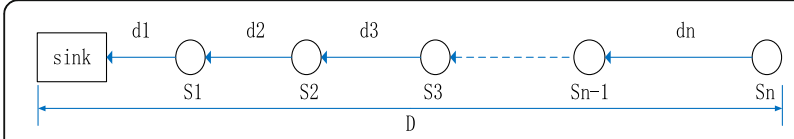

Fig. 1 Illustration of linear network deployment
Table 1 Network parameters

\begin{tabular}{lll}
\hline Parameter & Value & Value \\
\hline$T$ & Transmission period & $100 \mathrm{~ms}$ \\
$T_{\text {start }}$ & The time of transient mode & $510^{-6} \mathrm{~S}$ \\
$\eta$ & The power of amplifier & 0.35 \\
$k$ & The exponent order & 3 \\
$G_{1}$ & The gain factor & $10^{3}$ \\
$M_{1}$ & The link margin & $10^{4}$ \\
$P_{\text {circuit }}$ & The power of circuit & $140.7 \mathrm{~mW}$ \\
$P_{\text {syn }}$ & The power of frequency synthesizer & $50 \mathrm{~mW}$ \\
$\sigma^{2}$ & The AWGN power spectral density & $3.98110^{-21}$ \\
$B$ & The channel bandwidth & $10^{4}$ \\
$L$ & The number of bits in a packet & $10^{3}$ \\
$N_{f}$ & The receiver noise figure & 10 \\
\hline
\end{tabular}

$$
\begin{aligned}
& E_{\text {totalMQAM }}=\frac{4}{3}(1+\beta) N_{f} \sigma^{2}(M-1) \operatorname{In}\left(\frac{4\left(1-\frac{1}{\sqrt{M}}\right)}{b P_{e}}\right) B \tau T G_{d} \\
&+\left(P_{\text {circuit }}\right) \tau T+2 P_{\text {syn }} T_{\text {start }}
\end{aligned}
$$

For MPSK, according to Eqs. (4)-(9), the energy consumed to transmit a packet when using MPSK technology is as:

$$
\begin{aligned}
E_{\text {total_MPSK }} & =2(1+\beta) N_{f} \sigma^{2}\left(\frac{\operatorname{In}\left(\frac{2}{b P_{e}}\right)}{\left(\sin \left(\frac{\pi}{M}\right)\right)^{2}}\right) B \tau T G_{d} \\
& +\left(P_{\text {circuit }}\right) \tau T+2 P_{\text {syn }} T_{\text {start }}
\end{aligned}
$$

The energy consumed to transmit a packet using MQAM or MPSK is defined as $E_{\text {total }}$. The energy consumption of nodes in linear network is shown in Theorem 1.

Theorem 1 Assume there are $n$ nodes (not include sink) in linear network, when $n$ nodes generate a data packet in a transmission period and transmit it to the sink, the energy consumption of node $i$ is:

$$
E_{i}=(n-i+1) E_{\text {total }}
$$

$E_{\text {total }}$ is the energy consumption to transmit a data packet using MQAM or MPSK, which is equal to (14) or (15).

Proof It is clear that the number of packet forwarded by the node $i$ is $n-i+1$, so the energy consumption of node $i$ is

$$
E_{i}=(n-i+1) E_{\text {total }}
$$

The following gives optimization methods for linear network under different modulation techniques. 


\subsubsection{The RDML scheme in MQAM technique}

This part focuses on the optimization for sensor network using the MQAM modulation technique. First, the network lifetime at a certain duty cycle is optimized by non-equidistant deployment of nodes, and the distance between nodes is as Theorem 2 .

Theorem 2 For linear network under MQAM modulation technique, to minimize the maximum energy consumption is to calculate a set of $d_{1}, d_{2}, d_{3}, \ldots, d_{n}$ at duty cycle $\tau$ to satisfy the following equation.

$$
\left\{\begin{array}{c}
n T_{1} d_{1}^{k}+E_{c}=(n-1) T_{1} d_{2}^{k} \\
(n-1) T_{1} d_{2}^{k}+E_{c}=(n-2) T_{1} d_{3}^{k} \\
\vdots \\
(n-i+1) T_{1} d_{i}^{k}+E_{c}=(n-i) T_{1} d_{i+1}^{k} \\
\vdots \\
2 T_{1} d_{n-1}^{k}+E_{c}=T_{1} d_{n}^{k} \\
\sum_{i=1}^{n} d_{i}=D
\end{array}\right.
$$

where $\quad T_{1}=\frac{4}{3}(1+\beta) N_{f} \sigma^{2}(M-1) \ln \left(\frac{4\left(1-\frac{1}{\sqrt{M}}\right)}{b P_{e}}\right) B G_{1} M_{1} T \tau$, $E_{c}=\left(\left(P_{\text {circuit }}\right) T \tau+2 P_{\text {syn }} T_{\text {start }}\right)$.

Proof If the energy consumption of all nodes is balanced, the maximum energy consumption in network is minimum. So the $E_{i}=E_{j}, i \neq j$ is required.

Reorganizing the above formula, then:

$$
\left\{\begin{array}{c}
E_{1}=E_{2} \\
E_{2}=E_{3} \\
\vdots \\
E_{n-1}=E_{n} \\
\sum d_{i}=D
\end{array}\right.
$$

Substitute (16) to the above formula, then:

$$
\left\{\begin{array}{c}
n T_{1} d_{1}^{k}+E_{c}=(n-1) T_{1} d_{2}^{k} \\
(n-1) T_{1} d_{2}^{k}+E_{c}=(n-2) T_{1} d_{3}^{k} \\
\vdots \\
(n-i+1) T_{1} d_{i}^{k}+E_{c}=(n-i) T_{1} d_{i+1}^{k} \\
\vdots \\
2 T_{1} d_{n-1}^{k}+E_{c}=T_{1} d_{n}^{k} \\
\sum_{i=1}^{n} d_{i}=D
\end{array}\right.
$$

So, according to Eq. (17), we can calculate a set of $d_{1}$, $d_{2}, d_{3}, \ldots, d_{n}$ as:

$$
d_{i+1}=\sqrt[k]{\frac{i E_{c}}{(n-i) T_{1}}+\frac{n}{n-i} d_{1}^{k}}
$$

Theorem 3 For non-equidistant linear network under $M Q A M$ modulation technique, there exists an optimal duty cycle to minimize the maximum energy consumption. The maximum energy consumption is shown as:

$$
\begin{aligned}
E(\tau) & =n T_{2}(1+\beta)(M-1) \operatorname{In}\left(\frac{4\left(1-\frac{1}{\sqrt{M}}\right)}{b P_{e}}\right) \tau T d_{1}^{3} \\
& +n\left(P_{\text {circuit }}\right) \tau T+2 n P_{\text {syn }} T_{\text {start }}
\end{aligned}
$$

where $T_{2}=2 N_{f} \sigma^{2} B G_{1} M_{1}$.

Proof From Eq. (18), if the length of the linear network is unchanged, the distance between the first node and the sink becomes smaller as the duty cycle increases.

The node with the highest energy consumption in the linear network is divided into two parts, which is the energy consumption of transmission as:

$$
E_{\mathrm{tr}}=n T_{2}(1+\beta)(M-1) \ln \left(\frac{4\left(1-\frac{1}{\sqrt{M}}\right)}{b P_{e}}\right) \tau T d_{1}{ }^{3}
$$

The other part is the energy consumption of the circuit as:

$$
E_{\text {circuit }}=n\left(P_{\text {circuit }}\right) \tau T+2 n P_{\text {syn }} T_{\text {start }}
$$

As the duty cycle of the sensor node increased, the modulation level will be reduced. Therefore, the energy consumption of transmission will gradually decrease and the energy consumption of circuit increases linearly with the duty cycle. Therefore, it is possible that the energy consumption of transmission is same as that of circuit. If the above two parts are same, there must be a minimum total energy consumption. Therefore, the problem translates into whether two function images can be equal in a same duty cycle.

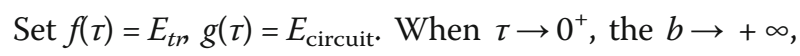
$M \rightarrow+\infty$, because the node's signal-to-noise ratio cannot be negative, so we require $\frac{4\left(1-\frac{1}{\sqrt{M}}\right)}{b P_{e}}>1$. When $\tau \rightarrow 0^{+}, \sqrt{M}$ is a large number, so the formula changes to $\frac{4}{b P_{e}}>1$. In other words, we need to meet $b<\frac{4}{P_{e}}$ and then the minimized duty cycle is $\tau<\frac{L P_{e}}{4 B T}$. It is clear that the duty cycle is a number close to zero because usually the sensor node BER is a very small number $\left(\leq 10^{3}\right)$, so $\sqrt{M}$ also which meets the above formula is a large number.

$\lim _{\tau \rightarrow \frac{L P_{e}}{4 B T} f}(\tau)=\lim _{b \rightarrow \rightarrow \frac{4}{P_{e}}} n T_{2}(1+\beta)(M-1) \ln \left(\frac{4\left(1-\frac{1}{\sqrt{M}}\right)}{b P_{e}}\right) \tau T d(\tau)^{3}$

The $\beta, d(\tau)$ is a constant which can be obtained from $b$ equals to $\frac{4}{P_{e}} ; M=2^{b}$ is a large number, and it is close to $\infty$. Therefore, in reorganizing the formula, we can get: 


$$
\lim _{b \rightarrow \frac{4}{P_{e}}}(M-1) \ln \left(\frac{4\left(1-\frac{1}{\sqrt{M}}\right)}{b P_{e}}\right)=\lim _{b \rightarrow \frac{4}{P_{e}}} \frac{\ln \left(\frac{4}{b P_{e}}\right)}{\frac{1}{\left(2^{b}-1\right)}}
$$

Using L' Hospital's rule

$$
\lim _{b \rightarrow \frac{4}{P_{e}}} \frac{\ln \left(\frac{4}{b P_{e}}\right)}{\frac{1}{\left(2^{b}-1\right)}}=\lim _{b \rightarrow \frac{4}{P_{e}}} \frac{\frac{1}{b}}{2^{b}\left(2^{b}-1\right)^{-2} \ln 2}=\lim _{b \rightarrow \frac{4}{P_{e}}} \frac{\left(2^{b}-1\right)^{2}}{b 2^{b} \ln 2}
$$

Obviously, $b$ is a large number. Thus, when $\tau \rightarrow \frac{L P_{e}}{4 B T}$, $f(\tau)$ is bound to infinity.

When $\tau \rightarrow 0^{+}$, the energy consumption of the circuit is as:

$$
\lim _{\tau \rightarrow 0^{+}} g(\tau)=2 n P_{\text {syn }} T_{\text {start }}
$$

Obviously, $f(\tau)>g(\tau)$.

When $\tau=1, f(1)=1.47 n T_{2} \ln \left(\frac{0.29}{P_{e}}\right) \operatorname{Td}(1)^{3}$.

$$
g(1)=n\left(P_{\text {circuit }}\right) T+2 n P_{\text {syn }} T_{\text {start }}
$$

Because $T_{2}$ is a small number $\left(<10^{-10}\right)$, we usually can get $g(1)>f(1)$.

Then, tiding up the above, when $\tau \rightarrow \frac{L P_{e}}{4 B T}, f(\tau)>g(\tau)$, and when $\tau=1, g(\tau)>f(\tau)$, and the above two functions in the interval $(0,1)$ is a continuous function. Thus, there are intersections between the two functions. So the maximum energy consumption have a minimized value.

The energy consumption of transmission and circuit in a linear network with $n=6, D=500 \mathrm{~m}$ is shown in Fig. 2. Obviously, there exists an intersection point and an optimal duty cycle to minimize the maximized energy consumption.

Theorem 2 shows that the network lifetime can be maximized by non-equidistant deployment of nodes in a certain duty cycle. Theorem 3 shows that the network lifetime can be further increased by finding an optimal duty cycle so our goal $\min \left\{\max \left(E_{i}(\tau)\right)\right\}$ can be reached.

But it is impossible to completely balance the energy consumption in the network. Therefore, the nodes in the linear network have residual energy, so we can use the residual energy to increase the duty cycle of nodes (see in Theorem 4) and reduce the node's BER (see in Theorem 5). Therefore, this strategy can achieve the purpose of reducing the delay.

Theorem 4 For linear network, nodes are deployed equidistantly. The duty cycle of each node can be calculated as follows to reduce the delay of each node.

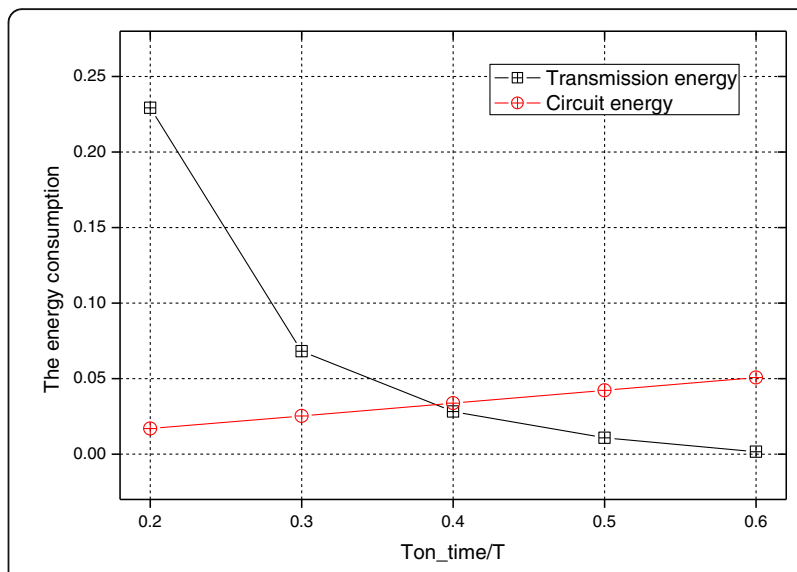

Fig. 2 The energy consumption of transmission and circuit (MQAM)

$$
\left\{\begin{array}{l}
E_{\text {total }}\left(\tau_{i}\right)=(1+\beta) P_{\mathrm{rx}} G_{d} \tau_{i} T+E_{c} \\
\text { s.t. }(n-i+1) E_{\text {total }}\left(\tau_{i}\right) \leq n E_{\text {total }}\left(\tau_{\text {opt }}\right)
\end{array}\right.
$$

where $P_{r x}$ is receiver power, $E_{c}=\left(P_{\text {circuit }}\right) \tau_{i} T+2 P_{\text {syn }} T_{\text {start }}$.

Proof It is clear that the node which is closest to the sink has the maximum energy consumption in linear network. Assume $E_{\text {total }}\left(\tau_{i}\right)$ is the energy consumption of transmitting a data packet. Because we hope the network lifetime to be as long as possible, the node with the largest energy consumption uses the optimal duty cycle, and the energy consumption of other nodes does not exceed the maximum energy consumption, so the network lifetime will not be reduced. So, according to (19), we can calculate the duty cycle of each node.

Theorem 5 Under MQAM modulation technique, the relationship between BER and duty cycle is as follows

$$
P_{e}=\frac{4\left(1-\frac{1}{\sqrt{M}}\right) B \tau T}{L e^{K_{1}}}
$$

where $K_{1}=\frac{3 P_{r x} \text { MQAM }}{4 B \sigma^{2}(M-1) N_{f}}$ and $M$ is up to the duty cycle.

Proof According to (8), the BER can be obtained from the duty cycle

$$
P_{e} \approx \frac{4}{b}\left(1-\frac{1}{\sqrt{M}}\right) e^{\left(-\frac{3}{M-1}\right) \frac{S N R}{2}}=\frac{4 B \tau T}{L}\left(1-\frac{1}{\sqrt{M}}\right) e^{\left(-\frac{3}{M-1}\right) \frac{S N R}{2}}
$$

And the signal-to-noise ratio can be obtained from the receiver power

$$
S N R=\frac{P_{\mathrm{rx} \_\mathrm{MQAM}}}{2 B N_{f} \sigma^{2}}
$$

So we can get:

$$
P_{e}=\frac{4\left(1-\frac{1}{\sqrt{M}}\right) B \tau T}{L e^{K_{1}}}
$$

where $M=2^{\frac{L}{B T T}}$ is up to the duty cycle. From this, the 
relationship between the BER and the duty cycle is obtained.

From Fig. 3, it can be seen that the BER will gradually decrease with the increase of the duty cycle.

The delay can be obtained from the BER by Eqs. (10) and (11). Obviously, as the BER decreases, the delay will also decrease, and in Theorem 5, the BER decreases as the duty cycle increases. So, the larger the duty cycle of the nodes, the smaller the delay. Therefore, this paper can reduce the delay by increasing the duty cycle of the node that is away from the sink. The following in Theorem 6 give the formula for the delay calculation under the optimization strategy of this paper.

Theorem 6 For linear network under MQAM modulation technique, the delay ratio of the optimization strategy in this paper to the ODCNP (optimal duty cycle of nodes policy) is given by:

$$
\phi=\frac{\sum_{i=1}^{n} \varpi\left(\tau_{i}\right)}{n \varpi\left(\tau_{\mathrm{opt}}\right)}
$$

where the delay can be computed by $\varpi(\tau)=$ $\left(1-\frac{4 B \tau T\left(1-\frac{1}{\sqrt{M}}\right) e^{-\frac{3 S N R}{2(M-1)}}}{L}\right)$ and $M=2^{\frac{L}{B \tau T}}$.

Proof In order to facilitate the calculation, the transmit power of each node is assumed same in network and the signal-to-noise ratio of each node will not change, so we can get:

$$
P_{e} \approx \frac{4}{b}\left(1-\frac{1}{\sqrt{M}}\right) e^{\left(-\frac{3}{M-1}\right) \frac{S N R}{2}}
$$

According to (14) and (15), combined with the above formula, we can get

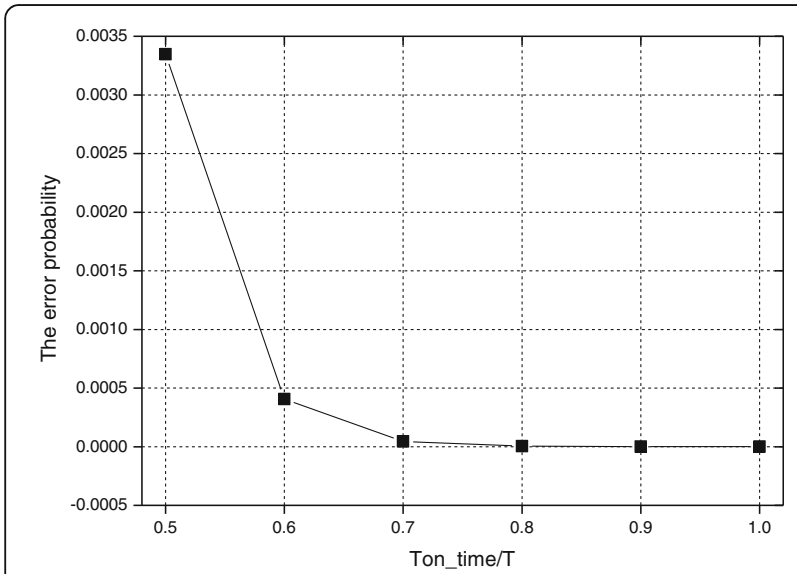

Fig. 3 The bit error rate under MQAM modulation

$$
\varpi(\tau)=\left(1-\frac{4 B \tau T\left(1-\frac{1}{\sqrt{M}}\right) e^{-\frac{3 S N R}{2(M-1)}}}{L}\right)^{L}
$$

In linear network, the data is sent to the sink at most via $n$ hops. Therefore, the maximum delay of the network is $n \varpi\left(\tau_{\text {opt }}\right)$ when the nodes are used for the optimal duty cycle. So the delay ratio of the optimization strategy in this paper to the previous strategy is given by

$$
\phi=\frac{\sum_{i=1}^{n} \varpi\left(\tau_{i}\right)}{n \varpi\left(\tau_{\mathrm{opt}}\right)}
$$

\subsubsection{The RDML scheme in MPSK technique}

Similar to the previous analysis, when the modulation technique is MPSK, the non-equidistant strategy is used to optimize the network lifetime and the distance between nodes is as Theorem 7 .

Theorem 7 For linear network under MPSK modulation technique, minimizing the maximized energy consumption is to calculate a set of $d_{1}, d_{2}, d_{3}, \ldots, d_{n}$ to satisfy the following equation:

$$
\left\{\begin{array}{c}
n T_{3} d_{1}^{k}+E_{c}=(n-1) T_{3} d_{2}^{k} \\
(n-1) T_{3} d_{2}^{k}+E_{c}=(n-2) T_{3} d_{3}^{k} \\
\vdots \\
(n-i+1) T_{3} d_{i}^{k}+E_{c}=(n-i) T_{3} d_{i+1}^{k} \\
\vdots \\
2 T_{3} d_{n-1}^{k}+E_{c}=T_{3} d_{n}^{k} \\
\sum_{i=1}^{n} d_{i}=D
\end{array}\right.
$$
where $\quad T_{3}=2(1+\beta) N_{f} \sigma^{2}\left(\frac{\ln \left(\frac{2}{b P_{e}}\right)}{\left(\sin \left(\frac{\pi}{M}\right)\right)^{2}}\right) B \tau T G_{1} M_{1}, E_{c}$ is
same as Theorem 2.

Proof Under the MPSK modulation technique, to minimize the maximum energy consumption of the network, the energy consumption of each node also needs to be balanced, so the equation is:

$$
\left\{\begin{array}{c}
E_{1}=E_{2} \\
E_{2}=E_{3} \\
\vdots \\
E_{n-1}=E_{n} \\
\sum d_{i}=D
\end{array}\right.
$$

Substitute (20) into the above formula, that is: 


$$
\left\{\begin{array}{c}
n T_{3} d_{1}^{k}+E_{c}=(n-1) T_{3} d_{2}^{k} \\
(n-1) T_{3} d_{2}^{k}+E_{c}=(n-2) T_{3} d_{3}^{k} \\
\vdots \\
(n-i+1) T_{3} d_{i}^{k}+E_{c}=(n-i) T_{3} d_{i+1}^{k} \\
\vdots \\
2 T_{3} d_{n-1}^{k}+E_{c}=T_{3} d_{n}^{k} \\
\sum_{i=1}^{n} d_{i}=D
\end{array}\right.
$$

Solving Eq. (21), we can calculate a set of $d_{1}, d_{2}, d_{3}, \ldots$, $d_{n}$ as follows:

$$
d_{i+1}=\sqrt[k]{\frac{i E_{c}}{(n-i) T_{3}}+\frac{n}{n-i} d_{1}^{k}}
$$

Theorem 8 For non-equidistant linear network under MPSK modulation technique, there exists an optimal duty cycle $\tau$, which minimizes the maximum energy consumption. The maximum energy consumption is:

$$
\begin{aligned}
E(\tau) & =n T_{4}(1+\beta) \operatorname{In}\left(\frac{2}{b P_{e}}\right) \tau T d_{1}^{3} /(\sin (\pi / M))^{2} \\
& +n\left(P_{\text {circuit }}\right) \tau T+2 n P_{\text {syn }} T_{\text {start }}
\end{aligned}
$$

where $T_{4}=2 N_{f} \sigma^{2} B G_{1} M_{1}$.

Proof According to Eq. (22), the distance between the first node and the sink decreases as the duty cycle increases.

The node with the highest energy consumption in linear network is divided into two parts, which is the energy consumption of transmission as:

$$
E_{t r}=n T_{4}(1+\beta) \ln \left(\frac{2}{b P_{e}}\right) \tau T d_{1}^{3} /(\sin (\pi / M))^{2}
$$

The other part is the energy consumption of circuit as:

$$
E_{\text {circuit }}=n\left(P_{\text {circuit }}\right) \tau T+2 n P_{\text {syn }} T_{\text {start }}
$$

Then, the problem is whether the two functions of the duty cycle have an intersection.

Set $f(\tau)=E_{t n} g(\tau)=E_{\text {circuit }}$. When $\tau \rightarrow 0^{+}$, the $b \rightarrow+\infty$, $M \rightarrow+\infty$. Because the signal-to-noise ratio cannot be negative, we require $\frac{2}{b P_{e}}>1$. In other words, we need to meet $b<\frac{2}{P_{e}}$, and then, the minimized duty cycle is $\tau$ $<\frac{L P_{e}}{2 B T}$. It is clear that the duty cycle is a number close to zero because usually the sensor node BER is a very small number $\left(\leq 10^{3}\right)$.

$\lim _{\tau \rightarrow \frac{L P_{e}}{2 B T}} f(\tau)=\lim _{b \rightarrow \frac{2}{P_{e}}} n T_{2}(1+\beta) \ln \left(\frac{2}{b P_{e}}\right) \tau T d(\tau)^{3} /\left(\sin \left(\frac{\pi}{M}\right)\right)^{2}$

The $\beta, d(\tau)$ is a constant which can be obtained from $b$ equals to $\frac{2}{P_{e}} ; M=2^{b}$ is a big number, and it is close to $\infty$. So the formula is converted into a type of $\frac{0}{0}$.

Replacing with equivalent infinitesimal: $\lim _{b \rightarrow \frac{2}{P_{e}}} \frac{\ln \left(\frac{2}{b P_{e}}\right)}{\left(\sin \left(\frac{\pi}{M}\right)\right)^{2}}=\lim _{b \rightarrow \frac{2}{P_{e}}} \frac{\ln \left(1+\frac{2-b P_{e}}{b P_{e}}\right)}{\left(\frac{\pi}{M}\right)^{2}}=\lim _{b \rightarrow \frac{2}{P_{e}}} \frac{\frac{2-b P_{e}}{b P_{e}}}{\frac{\pi^{2}}{2^{2 b}}}$

Using L' Hospital's rule

$$
\lim _{b \rightarrow \frac{2}{P_{e}}} \frac{\frac{2-b P_{e}}{\frac{b P_{e}}{\pi^{2}}}}{\frac{\pi^{2 b}}{2^{2}}}=\lim _{b \rightarrow \frac{2}{P_{e}}} \frac{2^{2 b}}{\pi^{2} b^{2} P_{e} \ln 2}
$$

Obviously, $b$ is a big number, so when $\tau \rightarrow \frac{L P_{e}}{2 B T}$, the $f(\tau) \rightarrow+\infty$.

When $\tau \rightarrow 0^{+}$, the energy consumption of the circuit is as:

$$
\lim _{\tau \rightarrow 0^{+}} g(\tau)=2 n P_{\text {syn }} T_{\text {start }}
$$

It is clear that $f(\tau)>g(\tau)$.

When $\tau=1, f(1)=1.47 n T_{2} \ln \left(\frac{1}{P_{e}}\right) \operatorname{Td}(1)^{3}$.

$$
g(1)=n\left(P_{\text {circuit }}\right) T+2 n P_{\text {syn }} T_{\text {start }}
$$

Because $T_{2}$ is a small number $\left(<10^{-10}\right)$, we usually can get $g(1)>f(1)$.

Then, tiding up the above formula, when $\tau \rightarrow \frac{L P_{e}}{2 B T}, f(\tau)>$ $g(\tau)$, and when $\tau=1, g(\tau)>f(\tau)$, and the above two functions in the interval $(0,1)$ is a continuous function. Thus, there is an intersection between the two functions. So the maximum energy consumption has a minimum value.

From Fig. 4, it can be seen that the transmission energy and the circuit energy exist as an intersection, so they have an optimal value. Theorem 7 is shown that the energy consumption of each node in linear network can be balanced by adjusting the distance between each node. Theorem 8 shows that the network has an optimal duty cycle to minimize the maximum energy consumption under MPSK modulation technique.

For MPSK modulation technique, the same as the above formula, some nodes have residual energy. Because the difference of MQAM and MPSK is the calculation of the signal-to-noise ratio, according to Eq. (23), we also can compute the duty cycle.

Theorem 9 Under MPSK modulation technique, the relationship of BER and duty cycle is as:

$$
P_{e}=\frac{2 B \tau T}{L e^{K_{2}}}
$$

where $K_{2}=\frac{P_{\mathrm{rxMPSK}}(\sin (\pi / M))^{2}}{2 B \sigma^{2} N_{f} G_{d}}$ and $M$ is up to the duty cycle.

Proof According to (9), the BER can be obtained from the signal-to-noise as: 


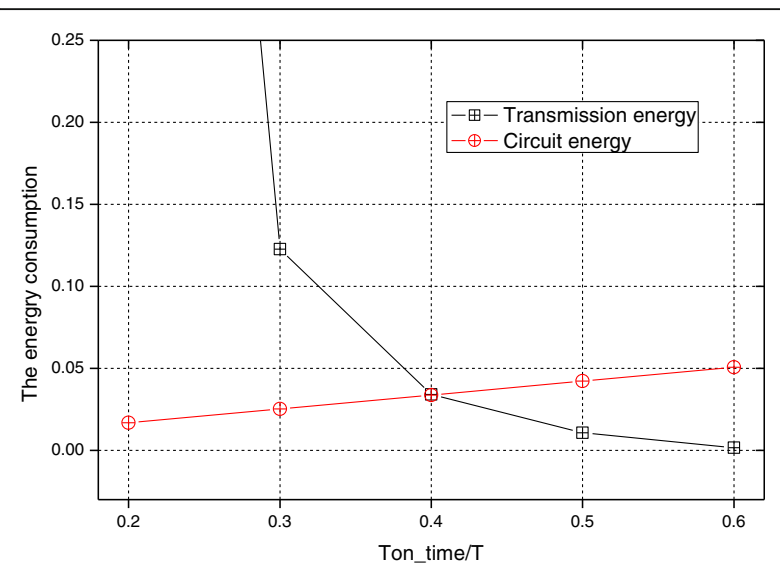

Fig. 4 The energy consumption of transmission and circuit (MPSK)

$$
P_{e} \approx \operatorname{erfc}\left(\sqrt{S N R} \sin \left(\frac{\pi}{2 M}\right)\right)
$$

And the signal-to-noise can be obtained from the receiver power

$$
S N R=\frac{P_{\text {rx_MPSK }}}{2 B N_{f} \sigma^{2}}
$$

Substitute the signal-to-noise ratio into the BER, so we can get:

$$
P_{e}=\frac{2 B \tau T}{L e^{K_{2}}}
$$

where $M=2^{\frac{L}{B \tau T}}$.

From Fig. 5, it can be seen that the BER will gradually decrease with the increase of the duty cycle when adopting the MPSK modulation technique.

From Eqs. (10) and (11), the delay will decrease with the duty cycle increase. Theorem 9 gives the relationship between the BER and the duty cycle in MPSK modulation technique, and the BER will decrease with the duty cycle increase. Therefore, the delay will decrease with the duty cycle increase. Therefore, the delay of network can be reduced by increasing the duty cycle of node which is far away from sink. Theorem 10 gives the calculation formula as follows.

Theorem 10 For linear network under MPSK modulation technique, the delay ratio of the optimization strategy in this paper and ODCNP is as follows:

$$
\phi=\frac{\sum_{i=1}^{n} \varpi\left(\tau_{i}\right)}{n \varpi\left(\tau_{\mathrm{opt}}\right)}
$$

where the delay ${ }_{L}$ can be computed by: $\varpi(\tau)=$ $\left(1-\frac{2 B \tau T e^{-(\sin (\pi / M))^{2} \cdot S N R}}{L}\right)$, and $M=2^{\frac{L}{B r T}}$.

Proof In order to facilitate the calculation, the transmit power of each node is assumed same in network and the signal-to-noise of each node will not change, so we can get:

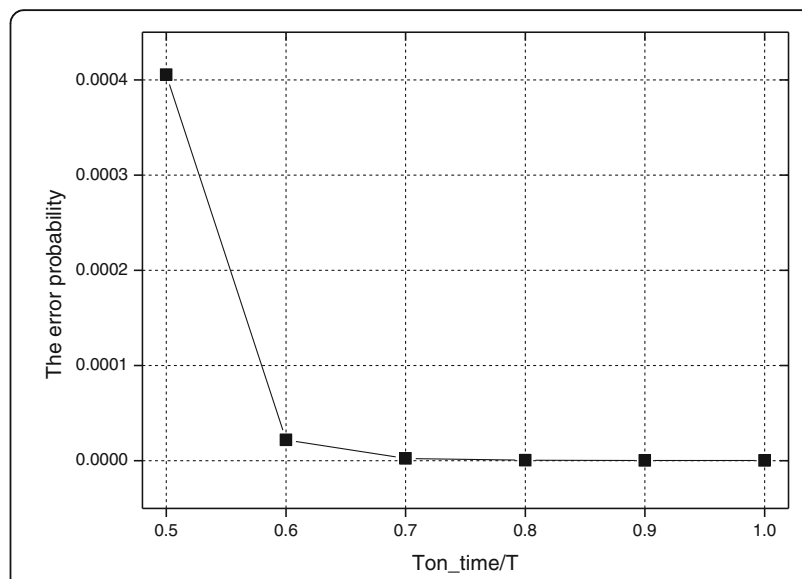

Fig. 5 The bit error rate under MPSK modulation

$$
P_{e} \approx \frac{2 e^{-(\sin (\pi / M))^{2} \cdot S N R}}{b}
$$

According to (10) and (11), combined with the above formula, we can get

$$
\varpi(\tau)=\left(1-\frac{2 B \tau T e^{-(\sin (\pi / M))^{2} \cdot S N R}}{L}\right)^{L}
$$

For linear network, the data is sent to the sink at most via $n$ hops. Therefore, the maximized delay of the network is $n \varpi\left(\tau_{\text {opt }}\right)$ when the nodes are used for the optimal duty cycle. So, the delay ratio of the optimization strategy in this paper to the previous strategy is given by

$$
\phi=\frac{\sum_{i=1}^{n} \varpi\left(\tau_{i}\right)}{n \varpi\left(\tau_{\mathrm{opt}}\right)}
$$

\subsection{The RDML scheme for Grid network}

Another network studied in this paper is the Grid network. The Grid network has applications in many scenarios $[41,62]$. The Grid network is a two-dimensional network. The deployment of nodes in the network is at the intersection of rows and columns, the sink is deployed at the intersection of the bottom leftmost row and column, and the sink is only connected with $S_{1,1}$. The network model is as follows:

Figure 6 is a $n \times n$ Grid network model. In a data collection round, the nodes except the sink generate a data packet. Because each node can only transmit to the left or down with the same probability, we can calculate the number of packets forwarded for each node as in Theorem 11.

Theorem 11 For Grid network, the data packets forwarded by each node is as follows: 


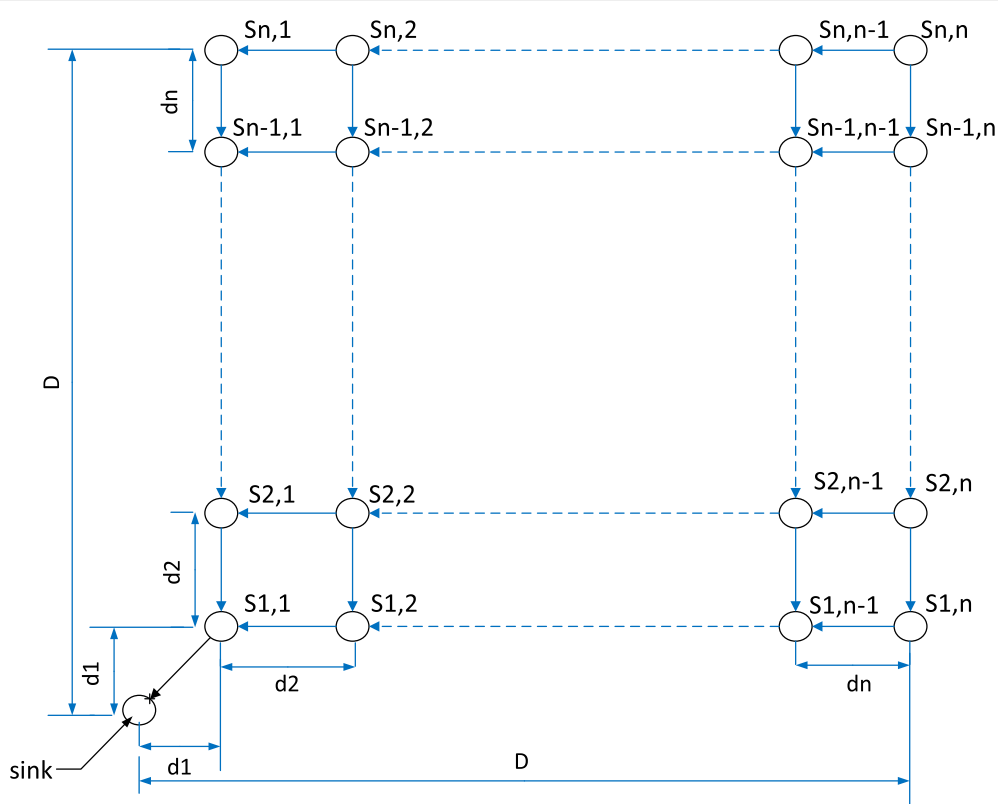

Fig. 6 Illustration of Grid network deployment

$$
\left\{\begin{array}{cc}
D_{n, n}=1 & \\
D_{n, j}=1+\frac{1}{2} D_{n, j+1} & (1 \leq j \leq n-1) \\
D_{i, 1}=1+D_{i+1,1}+\frac{1}{2} D_{i, 2} & (1 \leq i \leq n-1) \\
D_{i, j}=1+\frac{1}{2}\left(D_{i+1, j}+D_{i, j+1}\right) & (1<i, j<n) \\
D_{i, j}=D_{j, i} & (i \neq j)
\end{array}\right.
$$

Proof First, we analyze the nodes in the $n$th row. Since $D_{n, n}=1$ and each node is forwarding to the left or down with the same probability, we can get:

$$
D_{n, j}=1+\frac{1}{2} D_{n, j+1} \quad 1 \leq j<n
$$

Then, in analyzing the node in $(n-1)$ th row, we can get:

$$
\left\{\begin{array}{c}
D_{n, n-1}=1+\frac{1}{2} D_{n, n} \\
D_{n-1, j}=1+\frac{1}{2}\left(D_{n-1, j+1}+D_{n, j}\right) \quad 2 \leq j<n \\
D_{n-1,1}=1+D_{n, 1}+\frac{1}{2} D_{n-1,2}
\end{array}\right.
$$

And so on, reorganizing the above formula, we can get:

$$
\left\{\begin{array}{cc}
D_{n, n}=1 & \\
D_{n, j}=1+\frac{1}{2} D_{n, j+1} & (1 \leq j \leq n-1) \\
D_{i, 1}=1+D_{i+1,1}+\frac{1}{2} D_{i, 2} & (1 \leq i \leq n-1) \\
D_{i, j}=1+\frac{1}{2}\left(D_{i+1, j}+D_{i, j+1}\right) & (1<i, j<n) \\
D_{i, j}=D_{j, i} & (i \neq j)
\end{array}\right.
$$

Theorem 12 For Grid network, the node that is forwarding the most data packets must be in the first row or in the first column.

Proof In analyzing the nodes in the first row, the number of packets forwarded is:

$$
\left\{\begin{array}{c}
D_{i, 1}=1+D_{i+1,1}+\frac{1}{2} D_{i, 2} \quad 1 \leq i \leq n-1 \\
D_{n, 1}=1+\frac{1}{2} D_{n, 2}
\end{array}\right.
$$

In analyzing the nodes in the second row, the number of packets forwarded is:

$$
\left\{\begin{array}{c}
D_{i, 2}=1+\frac{1}{2}\left(D_{i, 3}+D_{i+1,2}\right) \quad 1 \leq i \leq n-1 \\
D_{n, 2}=1+\frac{1}{2} D_{n, 3}
\end{array}\right.
$$

In substituting $D_{n, 2}$ into (29), we can get:

$$
D_{n, 1}=\frac{3}{2}+\frac{1}{4} D_{n, 3}
$$

To prove $D_{n, 1}>D_{n, 2}$ is equivalent to prove $D_{n, 1}-$ $D_{n, 2}>0$

$$
\frac{1}{2}-\frac{1}{4} D_{n, 3}>0
$$

So, we require $D_{n, 3}<2$.

The number of data packets in the $n$th row can be rewritten as: 


$$
D_{n, j}=2-\left(\frac{1}{2}\right)^{n-j} \quad 1 \leq j \leq n-1
$$

So the number must be less than 2 . Thus, $D_{n, 1}>D_{n, 2}$.

Then, by the $n$th row derived to the $(n-1)$ th row, it is easy to know $D_{n-1,1}>D_{n-1,2}$.

And so on, get $D_{i, 1}>D_{i, 2}$.

Therefore, the nodes in the first row have the largest forwarding number of data packets. The same can also prove for the nodes in the first column.

\subsubsection{The RDML scheme in MQAM technique}

This section focuses on optimization for Grid network using MQAM modulation technique. From Theorem 12, the first column node has the most data packets to forward, so the first column node has the highest energy consumption. Because the network lifetime is determined by the nodes with the largest energy consumption, the first column is needed to be considered. Theorem 13 gives the energy consumption of the first column nodes.

Theorem 13 For Grid network under MQAM modulation technique, the energy consumption of the first column nodes is as:

$$
\left\{\begin{array}{l}
E_{i, 1}=D_{i, 1}\left(T_{1} d_{i}^{3}+E_{c}\right) \quad(1<i \leq n) \\
E_{1,1}=D_{1,1}\left(T_{1}\left(\sqrt{2} d_{1}\right)^{3}+E_{c}\right) \quad(i=1)
\end{array}\right.
$$

where $T_{1}=\frac{4}{3}(1+\beta) N_{f} \sigma^{2}(M-1) \ln \left(\frac{4\left(1-\frac{1}{\sqrt{M}}\right)}{b P_{e}}\right) B G_{1} M_{1} \tau T$.

Proof From Theorem 11, the number of data packets forwarded by the node $S_{i, 1}$ is $D_{i, 1}$. Considering the energy consumption of transmitting a data packet is $E_{\text {- }}$ total, thus, the energy consumption of node $S_{i, 1}$ is as:

$$
E_{i, 1}=D_{i, 1} E_{\text {total }}
$$

Substitute (20) into (31), and the transmission distance of node $S_{1,1}$ is different from the other node, so we can get:

$$
\left\{\begin{array}{l}
E_{i, 1}=D_{i, 1}\left(T_{1} d_{i}^{3}+E_{c}\right) \quad(1<i \leq n) \\
E_{1,1}=D_{1,1}\left(T_{1}\left(\sqrt{2} d_{1}\right)^{3}+E_{c}\right) \quad(i=1)
\end{array}\right.
$$

Theorem 14 For Grid network under MQAM modulation technique, minimizing the maximum energy consumption is to calculate a set of $d_{1}, d_{2}, d_{3}, \ldots, d_{n}$ to satisfy the following equation:

$$
\left\{\begin{array}{c}
D_{1,1} T_{1}\left(\sqrt{2} d_{1}\right)^{3}+D_{1,1} E_{c}=D_{2,1} T_{1} d_{2}^{3}+D_{2,1} E_{c} \\
D_{2,1} T_{1} d_{2}^{3}+D_{2,1} E_{c}=D_{3,1} T_{1} d_{3}^{3}+D_{3,1} E_{c} \\
\vdots \\
D_{i, 1} T_{1} d_{i}^{3}+D_{i, 1} E_{c}=D_{i+1,1} T_{1} d_{i+1}^{3}+D_{i+1,1} E_{c} \\
\vdots \\
D_{n-1,1} T_{1} d_{n-1}^{3}+D_{n-1,1} E_{c}=D_{n, 1} T_{1} d_{n}^{3}+D_{n, 1} E_{c} \\
\sum_{i=1}^{n} d_{i}=D
\end{array}\right.
$$

where $T_{1}$ is the same as Theorem 11 .

Proof For Grid network under MQAM modulation technique, minimizing the maximum energy consumption is needed to meet:

$$
\left\{\begin{array}{c}
E_{i, 1}=E_{i, 2} \\
E_{i, 2}=E_{i, 3} \\
\vdots \\
E_{i, n-1}=E_{i, n} \\
\sum d_{i}=D
\end{array}\right.
$$

In substituting (29) in the above formula, we can get:

$$
\left\{\begin{array}{c}
D_{1,1} T_{1}\left(\sqrt{2} d_{1}\right)^{3}+D_{1,1} E_{c}=D_{2,1} T_{1} d_{2}^{3}+D_{2,1} E_{c} \\
D_{2,1} T_{1} d_{2}^{3}+D_{2,1} E_{c}=D_{3,1} T_{1} d_{3}^{3}+D_{3,1} E_{c} \\
\vdots \\
D_{i, 1} T_{1} d_{i}^{3}+D_{i, 1} E_{c}=D_{i+1,1} T_{1} d_{i+1}^{3}+D_{i+1,1} E_{c} \\
\vdots \\
D_{n-1,1} T_{1} d_{n-1}^{3}+D_{n-1,1} E_{c}=D_{n, 1} T_{1} d_{n}^{3}+D_{n, 1} E_{c} \\
\sum_{i=1}^{n} d_{i}=D
\end{array}\right.
$$

In solving (32), we can get a set of $d_{1}, d_{2}, d_{3}, \ldots, d_{n}$ as follows:

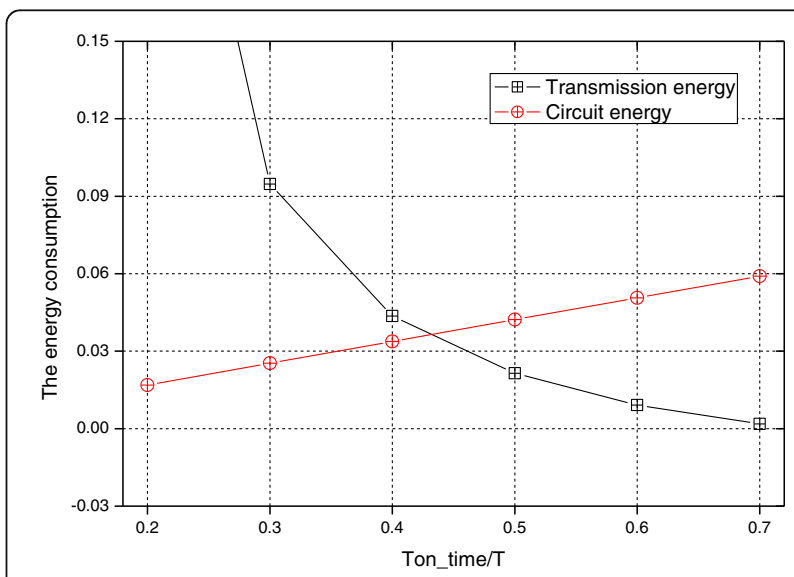

Fig. 7 The energy consumption of transmission and circuit at $S_{1}$, (MQAM) 


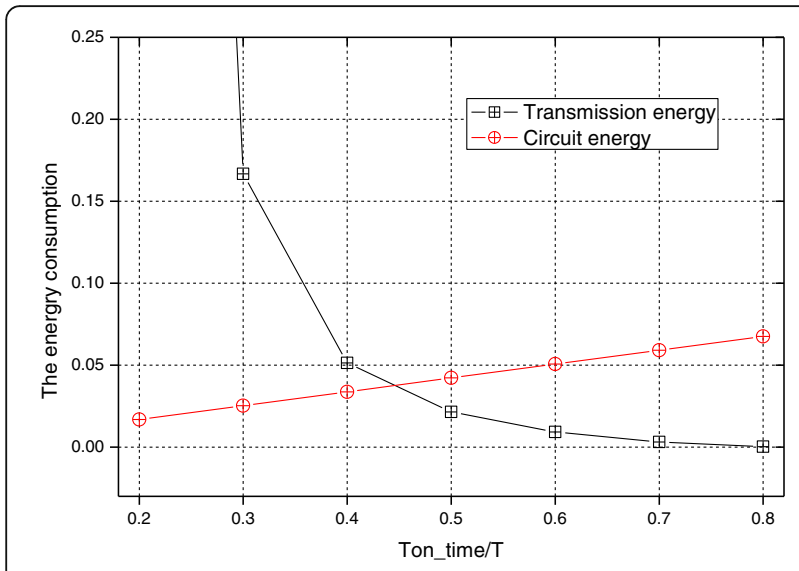

Fig. 8 The energy consumption of transmission and circuit at $s_{1}$, 1 (MPSK)

$$
d_{i+1}=\sqrt[3]{\frac{D_{1,1}}{D_{i+1,1}}\left(\sqrt{2} d_{1}\right)^{3}+\frac{\left(D_{1,1}-D_{i+1,1}\right) E_{c}}{D_{i+1,1} T_{1}}}
$$

Theorem 15 For Grid network under MQAM modulation technique, there exists an optimal duty cycle to maximize the network lifetime. The maximum energy consumption in the Grid network is as:

$$
\begin{aligned}
E(\tau) & =D_{1,1} T_{2}(1+\beta)(M-1) \operatorname{In}\left(\frac{4\left(1-\frac{1}{\sqrt{M}}\right)}{b P_{e}}\right) \tau T d_{1}^{3} \\
& +D_{1,1}\left(P_{\text {circuit }}\right) \tau T+2 D_{1,1} P_{\text {syn }} T_{\text {start }}
\end{aligned}
$$

where $T_{2}=\frac{4}{3} N_{f} \sigma^{2} B G_{1} M_{1}$.

Proof In the Grid network, when the row (column) length is unchanged, according to (33), the length of $d_{1}$ will decrease with the duty cycle increase.

The node with the highest energy consumption in the Grid network is divided into two parts, which is the energy consumption of transmission as:

Table 2 The unequal distance of nodes

\begin{tabular}{lllllll}
\hline$d$ & $d_{1}$ & $d_{2}$ & $d_{3}$ & $d_{4}$ & $d_{5}$ & $d_{6}$ \\
$\tau$ & & & & & & \\
\hline 0.2 & 63.98 & 68.27 & 73.83 & 81.59 & 93.76 & 118.6 \\
0.25 & 62.95 & 67.57 & 73.50 & 81.66 & 94.34 & 119.9 \\
0.3 & 61.36 & 66.52 & 73.03 & 81.83 & 95.27 & 122.0 \\
0.35 & 59.09 & 65.09 & 72.42 & 82.10 & 96.56 & 124.7 \\
0.4 & 56.03 & 63.26 & 71.73 & 82.54 & 98.26 & 128.2 \\
0.45 & 51.96 & 61.04 & 71.02 & 83.21 & 100.4 & 132.4 \\
0.5 & 46.55 & 58.50 & 70.42 & 84.24 & 103.1 & 137.2 \\
0.55 & 39.05 & 55.81 & 70.16 & 85.77 & 106.3 & 142.9 \\
0.6 & 27.54 & 53.58 & 70.68 & 88.17 & 110.5 & 149.5 \\
0.65 & 5.33 & 54.10 & 73.41 & 92.49 & 116.5 & 158.1 \\
\hline
\end{tabular}

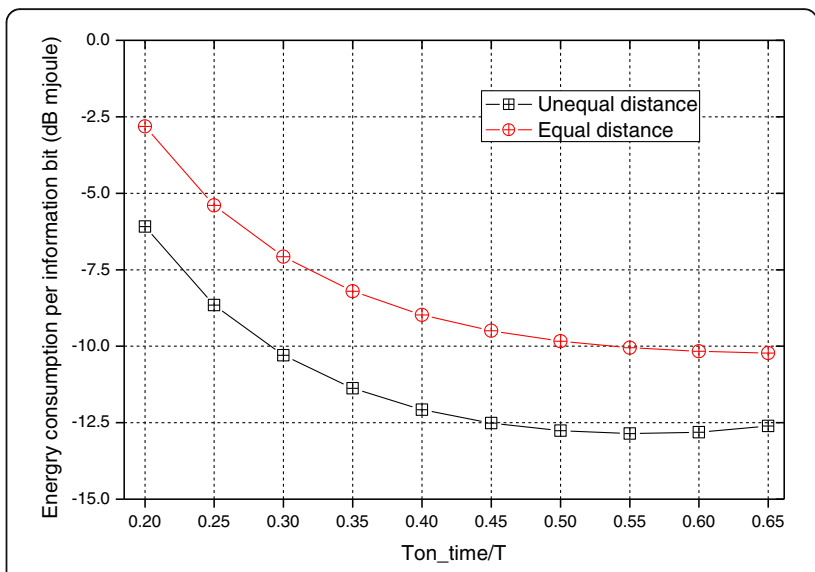

Fig. 9 The maximum energy consumption under UDNP and EDNP (MQAM)

$$
E_{\text {tr }}=D_{1,1} T_{2}(1+\beta)(M-1) \ln \left(\frac{4\left(1-\frac{1}{\sqrt{M}}\right)}{b P_{e}}\right) \tau T G_{1} M_{1} d_{1}{ }^{3}
$$

The other is the energy consumption of circuit as:

$$
E_{\text {circuit }}=D_{1,1}\left(P_{\text {circuit }}\right) \tau T+2 D_{1,1} P_{\text {syn }} T_{\text {start }}
$$

Because the energy consumption of these two parts and that of Theorem 3 has only the number of data packets different, and has the number of data packets constant, so similar with Theorem 3, we can prove that there are two curves intersecting and there is an optimal duty cycle to maximize the network.

Figure 7 shows the energy consumption of transmission and circuit at node $S_{1,1}$ in $n=6$, and the length of $\operatorname{row}($ column) is $D=500 \mathrm{~m}$. It can be seen they have an intersection.

Theorem 14 shows that there exists a set of $d_{1}, d_{2}, d_{3}$, $\ldots, d_{n}$ to ensure the energy consumption is balanced at the first row (column) nodes. Theorem 15 shows that

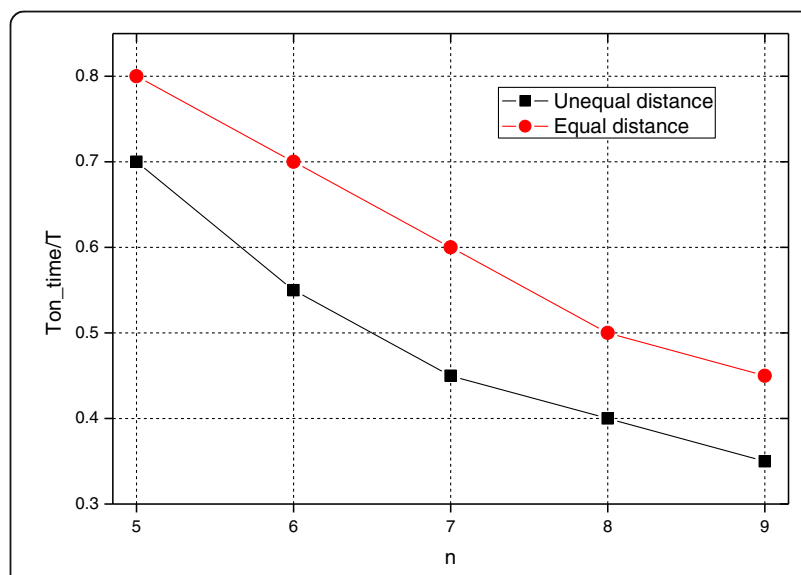

Fig. 10 The optimal duty cycle under UDNP and EDNP (MQAM) 


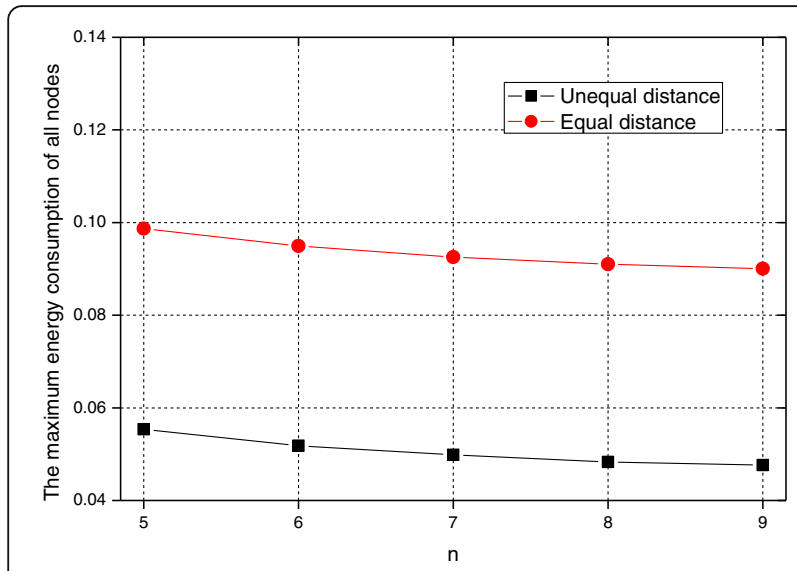

Fig. 11 The maximum energy consumption at optimal duty cycle under UDNP and EDNP (MQAM)

there is an optimal duty cycle to minimize the maximum energy consumption.

The same as the linear network, because the energy consumption of nodes is completely balanced which is very difficult, a strategy is used to increase the duty cycle of nodes with lower energy consumption in an equidistant deployed network, thereby reducing the delay. Theorem 16 gives the calculation method of the duty cycle of each node in the Grid network.

Theorem 16 For the Grid network, the nodes are deployed equidistantly and the duty cycle of each node is calculated as follows to reduce the delay of each node.

$$
\left\{\begin{array}{l}
E_{\text {total }}\left(\tau_{i, j}\right)=(1+\beta) P_{\mathrm{rx}} G_{d} \tau_{i, j} T+E_{c} \\
\text { s.t. } \quad D_{i, j} E_{\text {total }}\left(\tau_{i, j}\right) \leq D_{1,1} E_{\text {total }}\left(\left(\tau_{\text {opt }}\right)\right)
\end{array}\right.
$$

where $P_{r x}$ is the receiver power and $E_{c}=\left(\left(P_{\text {circuit }}\right) T \tau+\right.$ $\left.2 P_{\text {syn }} T_{\text {start }}\right)$.

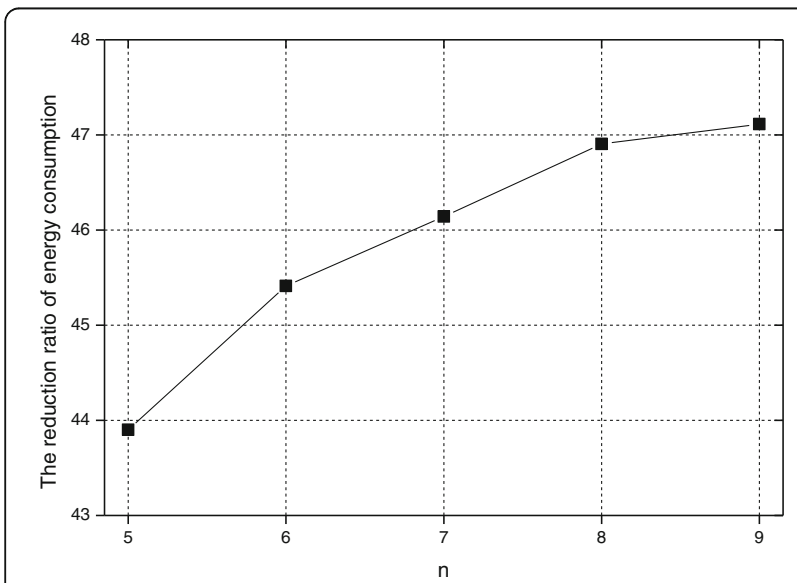

Fig. 12 The maximum energy consumption reduction ratio of UDNP vs EDNP (MQAM)

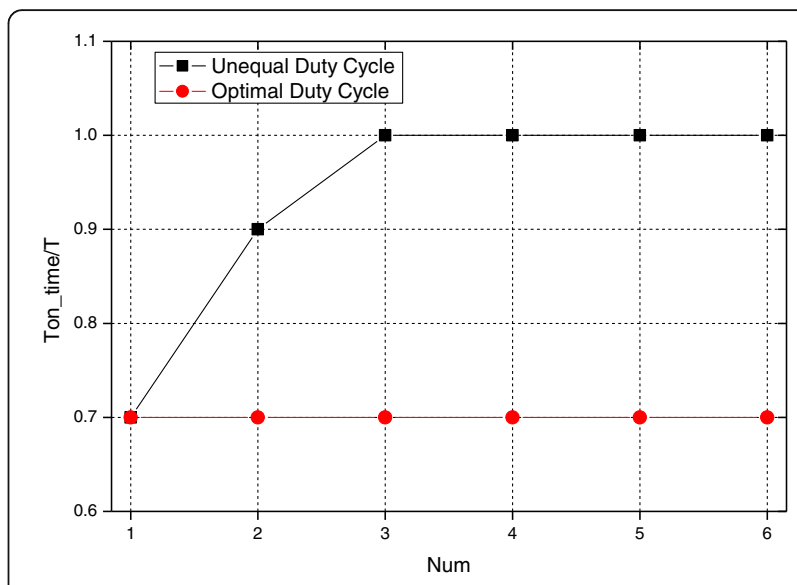

Fig. 13 The duty cycle used at each node in linear network (MQAM)

Proof According to Theorem 12, the node $S_{1,1}$ forwarded the largest data packet, so the maximum energy consumption is in this node. Therefore, node $S_{1,1}$ uses the optimal duty cycle to guarantee the maximum network lifetime. At the same time, the energy consumption of the other nodes in the network does not exceed that of node $S_{1,1}$ to guarantee the network lifetime. So according to Eq. (33), we can calculate the duty cycle of each node.

Theorem 17 For Grid network under MQAM modulation technique, the delay ratio of optimization strategy in this paper to the ODCNP is given by:

$$
\phi=\frac{\sum_{i=1}^{n} \varpi\left(\tau_{1, i}\right)+\sum_{i=2}^{n} \varpi\left(\tau_{i, n}\right)}{(2 n-1) \varpi\left(\tau_{\mathrm{opt}}\right)}
$$

where the delay $\varpi(\tau)$ and $M$ is the same as in Theorem 6.

Proof In order to facilitate the calculation, the transmit power of each node is assumed same in the Grid network, so the signal-to-noise ratio of each node will not change. So, we can get:

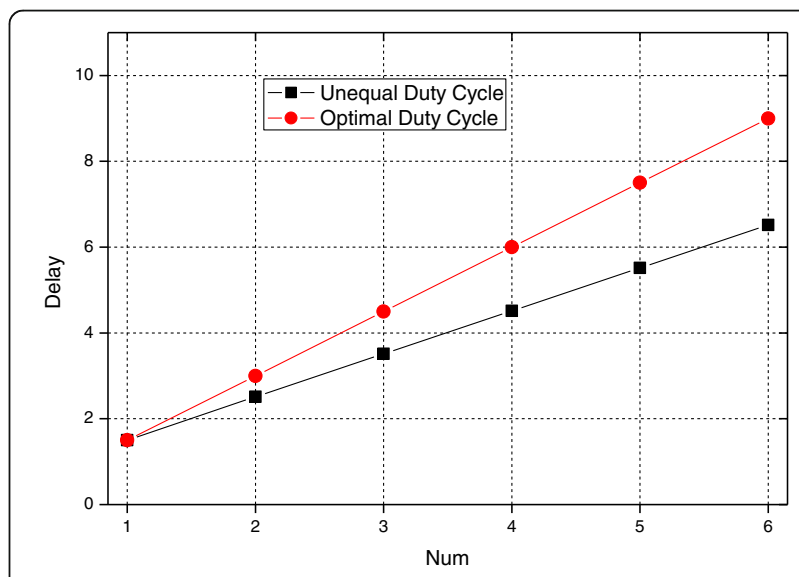

Fig. 14 The delay from each node in linear network to sink (MQAM) 


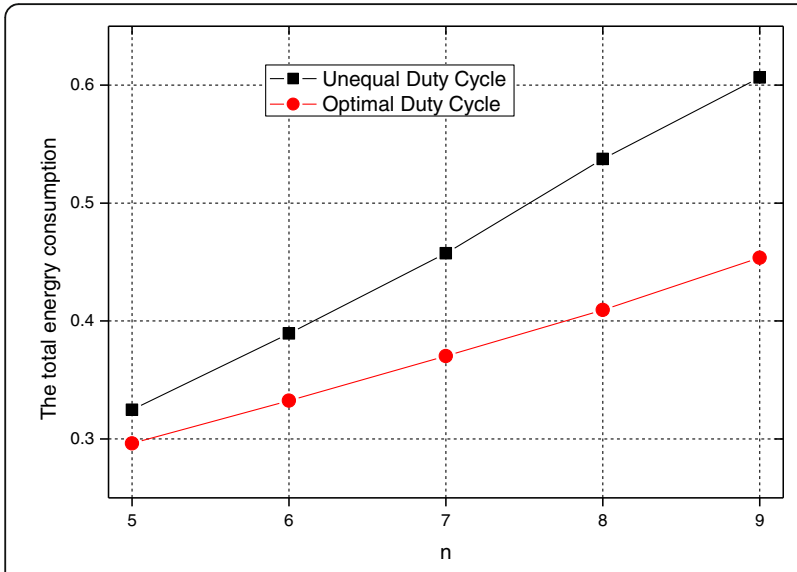

Fig. 15 The total energy consumption under UDCNP and ODCNP (MQAM)

$$
P_{e} \approx \frac{4}{b}\left(1-\frac{1}{\sqrt{M}}\right) e^{\left(-\frac{3}{M-1}\right) \frac{S N R}{2}}
$$

According to (14) and (15), combined with the above, we can get

$$
\varpi(\tau)=\left(1-\frac{4 B \tau T\left(1-\frac{1}{\sqrt{M}}\right) e^{-\frac{3 S N R}{2(M-1)}}}{L}\right)^{L}
$$

In the Grid network, the data is sent to the sink at most via $2 n-1$ hops, which is along the outermost edge of the network transmit to the sink. Therefore, the delay ratio is:

$$
\phi=\frac{\sum_{i=1}^{n} \varpi\left(\tau_{1, i}\right)+\sum_{i=2}^{n} \varpi\left(\tau_{i, n}\right)}{(2 n-1) \varpi\left(\tau_{\mathrm{opt}}\right)}
$$

Theorem 17 gives the delay formula in the Grid network, so the delay can be calculated in the Grid network under MQAM modulation technique.

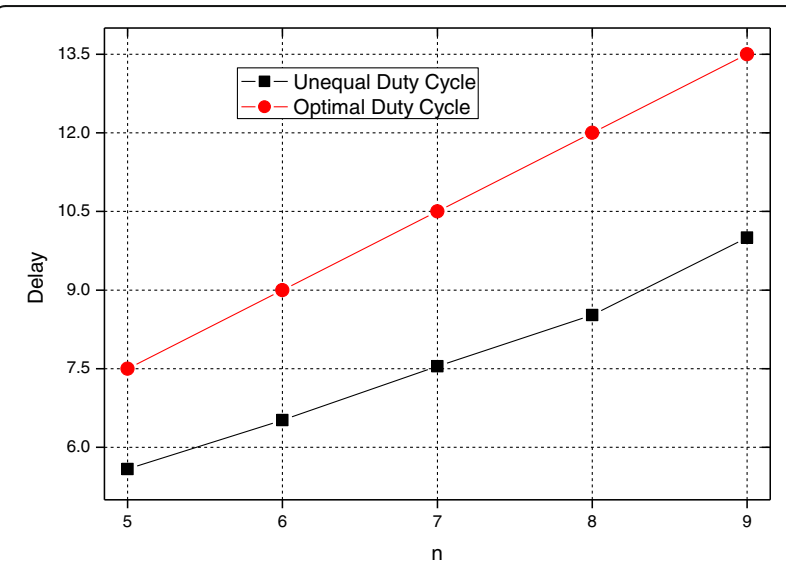

Fig. 16 The delay of UDCNP and ODCNP (MQAM)

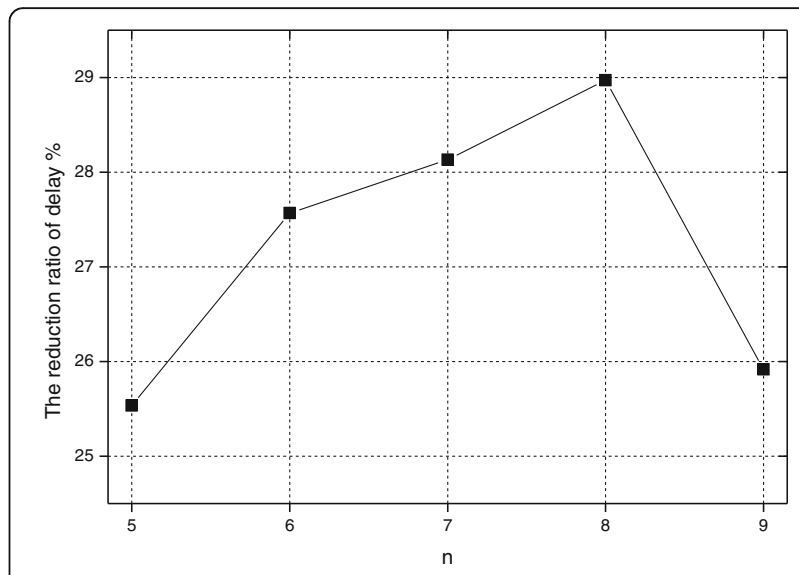

Fig. 17 The delay reduction of UDCNP vs ODCNP (MQAM)

\subsubsection{The RDML scheme in MPSK technique}

This section focuses on optimization for Grid network using MPSK modulation technique. First, Theorem 18 gives the energy consumption of the first column nodes under the MPSK modulation technique.

Theorem 18 For Grid network under MPSK modulation technique, the energy consumption of the first column is:

$$
\left\{\begin{array}{l}
E_{i, 1}=D_{i, 1}\left(T_{2} d_{i}^{3}+E_{c}\right) \quad(1<i \leq n) \\
E_{1,1}=D_{1,1}\left(T_{2}\left(\sqrt{2} d_{1}\right)^{3}+E_{c}\right) \quad(i=1)
\end{array}\right.
$$

where $T_{3}=2(1+\beta) N_{f} \sigma^{2}\left(\frac{\ln \left(\frac{2}{b P_{e}}\right)}{\left(\sin \left(\frac{\pi}{M}\right)\right)^{2}}\right) B \tau T G_{1} M_{1}$.

Proof Substitute (16) to (27), and the transmission distance of node $S_{1,1}$ is different from the other node, so we can get:

$$
\left\{\begin{array}{l}
E_{i, 1}=D_{i, 1}\left(T_{3} d_{i}^{3}+E_{c}\right) \quad(1<i \leq n) \\
E_{1,1}=D_{1,1}\left(T_{3}\left(\sqrt{2} d_{1}\right)^{3}+E_{c}\right) \quad(i=1)
\end{array}\right.
$$

Theorem 19 For Grid network under MPSK modulation technique, minimizing the maximum energy consumption

Table 3 The unequal distance of nodes under MPSK

\begin{tabular}{lllllll}
\hline$d$ & $d_{1}$ & $d_{2}$ & $d_{3}$ & $d_{4}$ & $d_{5}$ & $d_{6}$ \\
$\tau$ & & & & & & \\
\hline 0.2 & 64.55 & 68.65 & 74.02 & 81.53 & 93.41 & 117.79 \\
0.25 & 64.01 & 68.28 & 73.84 & 81.58 & 93.73 & 118.54 \\
0.3 & 62.74 & 67.43 & 73.43 & 81.68 & 94.46 & 120.20 \\
0.35 & 60.51 & 65.98 & 72.79 & 81.91 & 95.75 & 123.03 \\
0.4 & 57.16 & 63.92 & 71.97 & 82.36 & 97.63 & 126.96 \\
0.45 & 52.56 & 61.35 & 71.11 & 83.10 & 100.08 & 131.78 \\
0.5 & 46.54 & 58.50 & 70.42 & 84.23 & 103.05 & 137.24 \\
0.55 & 38.68 & 55.70 & 70.16 & 85.85 & 106.49 & 143.13 \\
0.6 & 27.80 & 53.60 & 70.66 & 88.11 & 110.43 & 149.40 \\
0.65 & 10.53 & 53.62 & 72.67 & 91.51 & 115.26 & 156.41 \\
\hline
\end{tabular}




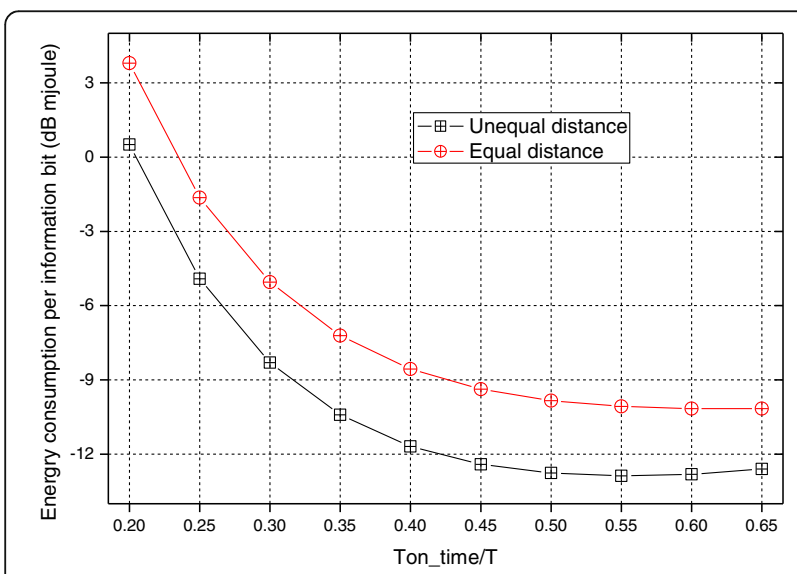

Fig. 18 The maximum energy consumption under UDNP and EDNP (MPSK)

is to calculate a set of $d_{1}, d_{2}, d_{3}, \ldots, d_{n}$ to satisfy the following equation

$$
\left\{\begin{array}{c}
D_{1,1} T_{3}\left(\sqrt{2} d_{1}\right)^{3}+D_{1,1} E_{c}=D_{2,1} T_{3} d_{2}^{3}+D_{2,1} E_{c} \\
D_{2,1} T_{3} d_{2}^{3}+D_{2,1} E_{c}=D_{3,1} T_{3} d_{3}^{3}+D_{3,1} E_{c} \\
\vdots \\
D_{i, 1} T_{3} d_{i}^{3}+D_{i, 1} E_{c}=D_{i+1,1} T_{3} d_{i+1}^{3}+D_{i+1,1} E_{c} \\
\vdots \\
D_{n-1,1} T_{3} d_{n-1}^{3}+D_{n-1,1} E_{c}=D_{n, 1} T_{3} d_{n}^{3}+D_{n, 1} E_{c} \\
\sum_{i=1}^{n} d_{i}=D
\end{array}\right.
$$

where $T_{3}$ is similar to Theorem 15 .

Proof For Grid network under MPSK modulation technique, minimizing the maximum energy consumption is needed to meet:

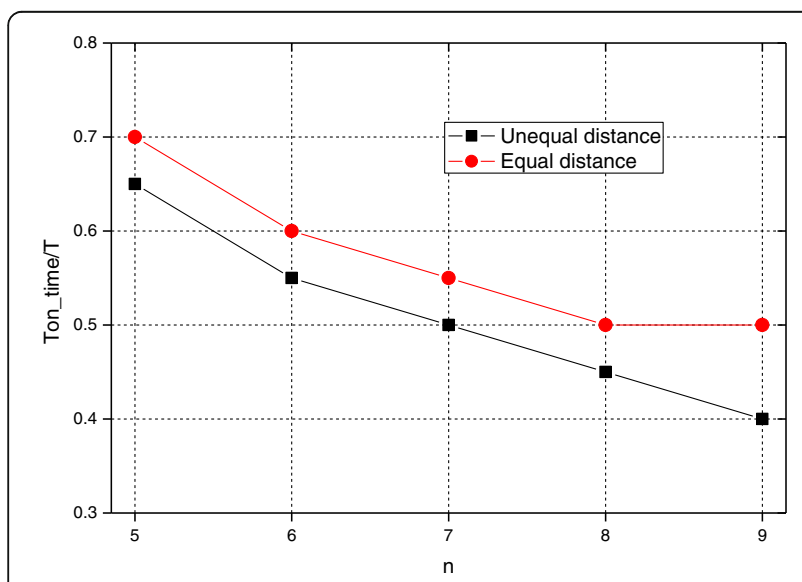

Fig. 19 The optimal duty cycle under UDNP and EDNP (MPSK)

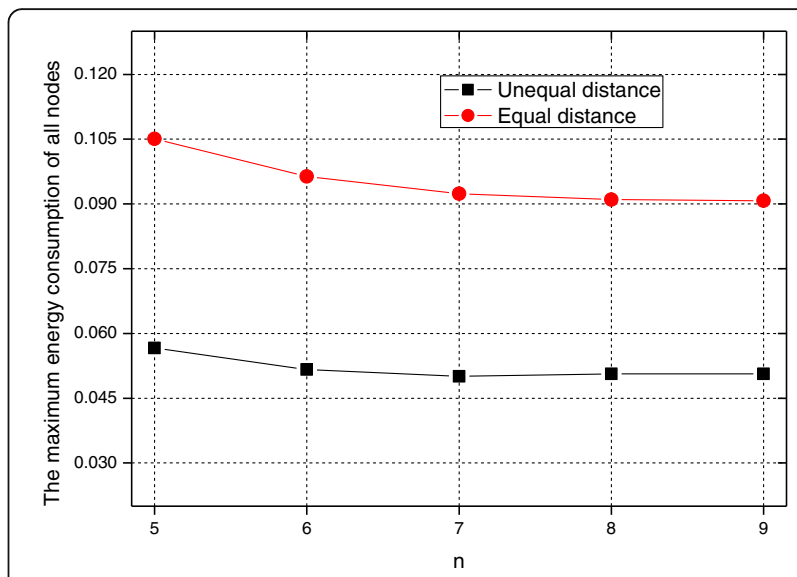

Fig. 20 The maximum energy consumption at optimal duty cycle under UDNP and EDNP (MPSK)

$$
\left\{\begin{array}{c}
E_{i, 1}=E_{i, 2} \\
E_{i, 2}=E_{i, 3} \\
\vdots \\
E_{i, n-1}=E_{i, n} \\
\sum d_{i}=D
\end{array}\right.
$$

In substituting (31) to the above formula, we can get:

$$
\left\{\begin{array}{c}
D_{1,1} T_{4}\left(\sqrt{2} d_{1}\right)^{3}+D_{1,1} E_{c}=D_{2,1} T_{4} d_{2}^{3}+D_{2,1} E_{c} \\
D_{2,1} T_{4} d_{2}^{3}+D_{2,1} E_{c}=D_{3,1} T_{4} d_{3}^{3}+D_{3,1} E_{c} \\
\vdots \\
D_{i, 1} T_{4} d_{i}^{3}+D_{i, 1} E_{c}=D_{i+1,1} T_{4} d_{i+1}^{3}+D_{i+1,1} E_{c} \\
\vdots \\
D_{n-1,1} T_{4} d_{n-1}^{3}+D_{n-1,1} E_{c}=D_{n, 1} T_{4} d_{n}^{3}+D_{n, 1} E_{c} \\
\sum_{i=1}^{n} d_{i}=D
\end{array}\right.
$$

In solving (32), we can get a set of $d_{1}, d_{2}, d_{3}, \ldots, d_{n}$ as follows:

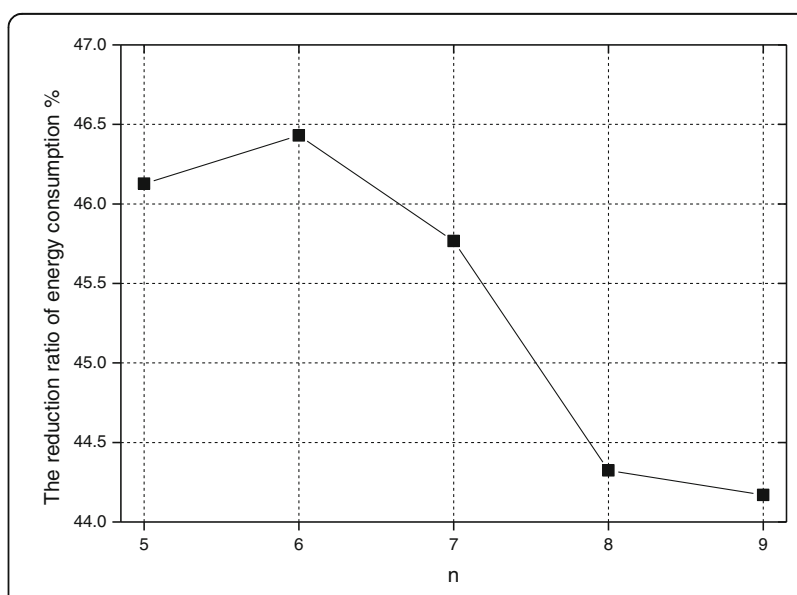

Fig. 21 The maximum energy consumption reduction ratio of UDNP vs EDNP (MPSK) 


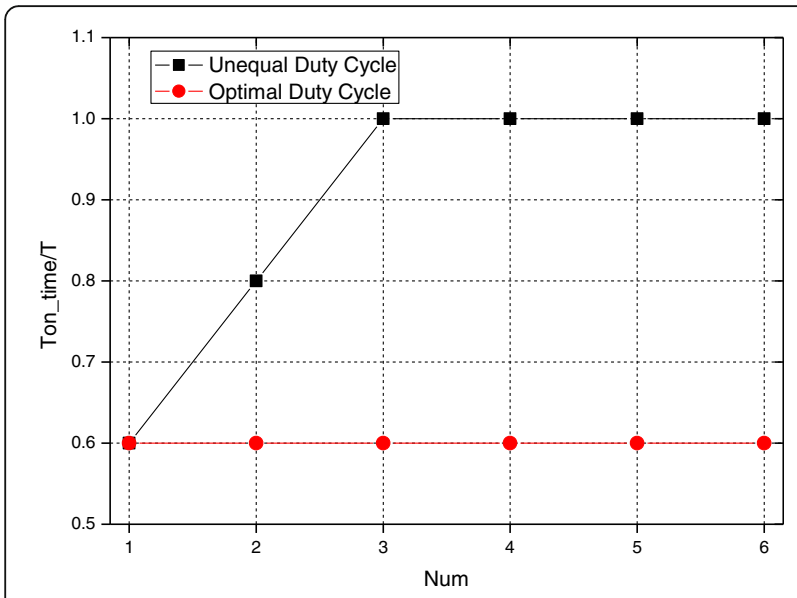

Fig. 22 The duty cycle used at each node in linear network (MPSK)

$$
d_{i+1}=\sqrt[3]{\frac{D_{1,1}}{D_{i+1,1}}\left(\sqrt{2} d_{1}\right)^{3}+\frac{\left(D_{1,1}-D_{i+1,1}\right) E_{c}}{D_{i+1,1} T_{3}}}
$$

Theorem 20 For Grid network under MPSK modulation technique, there exists an optimal duty cycle to maximize the network lifetime. The maximum energy consumption in Grid network is as:

$$
\begin{aligned}
E & =D_{1,1} T_{4}(1+\beta) \operatorname{In}\left(\frac{2}{b P_{e}}\right) \tau T d_{1}^{3} /(\sin (\pi / M))^{2} \\
& +D_{1,1}\left(P_{\text {circuit }}\right) \tau T+2 D_{1,1} P_{\text {syn }} T_{\text {start }}
\end{aligned}
$$

where $T_{4}=2 N_{f} \sigma^{2} B G_{1} M_{1}$.

Proof In Grid network, when the row (column) length is unchanged; according to (36), the length of $d_{1}$ will decrease with the duty cycle increase.

The node with the highest energy consumption in the Grid network is divided into two parts, which is the energy consumption of transmission as:

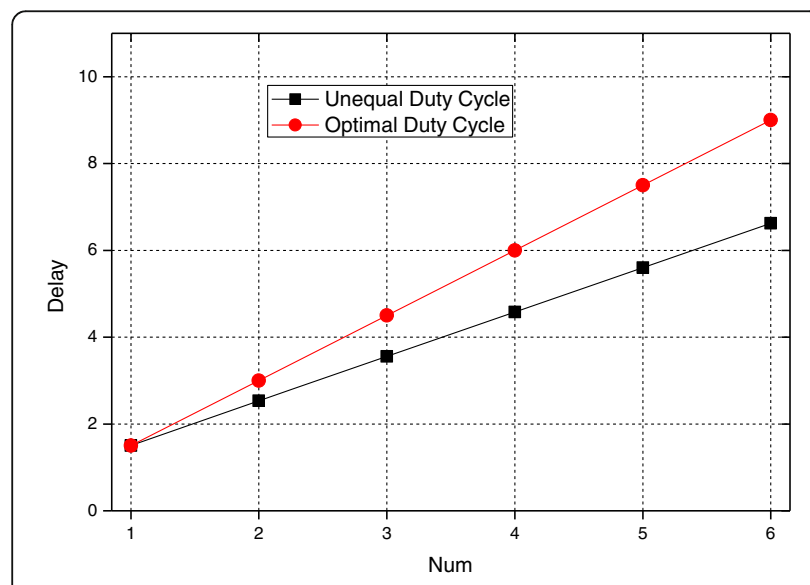

Fig. 23 The delay from each node in linear network to sink (MPSK)

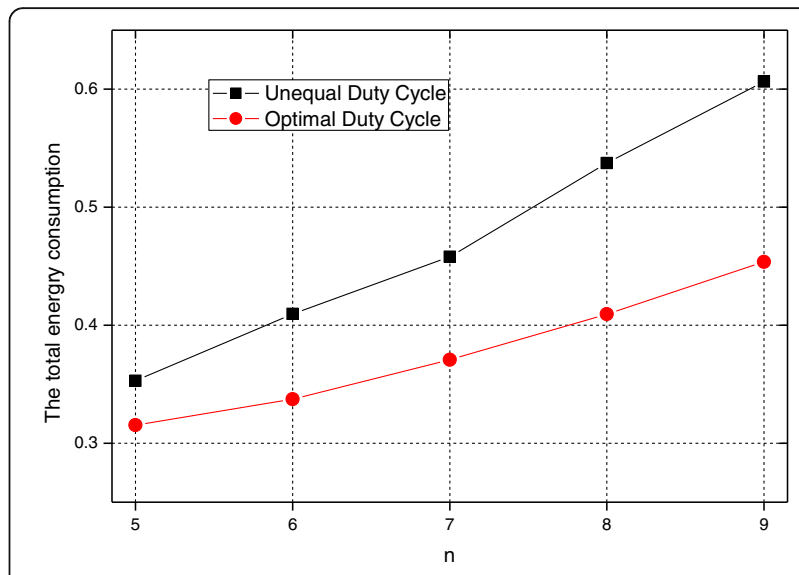

Fig. 24 The total energy consumption under UDCNP and OPCNP (MPSK)

$$
E_{\mathrm{tr}}=D_{1,1} T_{4}(1+\beta) \ln \left(\frac{2}{b P_{e}}\right) \tau T d_{1}^{3} /(\sin (\pi / M))^{2}
$$

The other is the energy consumption of the circuit as:

$$
E_{\text {circuit }}=D_{1,1}\left(P_{\text {circuit }}\right) \tau T+2 D_{1,1} P_{\text {syn }} T_{\text {start }}
$$

Similar with Theorem 7, we can prove that there are two curves intersecting and there is an optimal duty cycle to maximize the network.

From Fig. 8, we can know that they have an intersection, so there is an optimal duty cycle to maximize the network lifetime.

Theorem 19 shows that there exists a set of $d_{1}, d_{2}, d_{3}$, $\ldots, d_{n}$ to ensure the energy consumption is balanced at the first row (column) nodes, and Theorem 20 shows that there is an optimal duty cycle to maximize the network lifetime.

Theorem 16 also can get the calculation method of the duty cycle of each node in Grid network using the

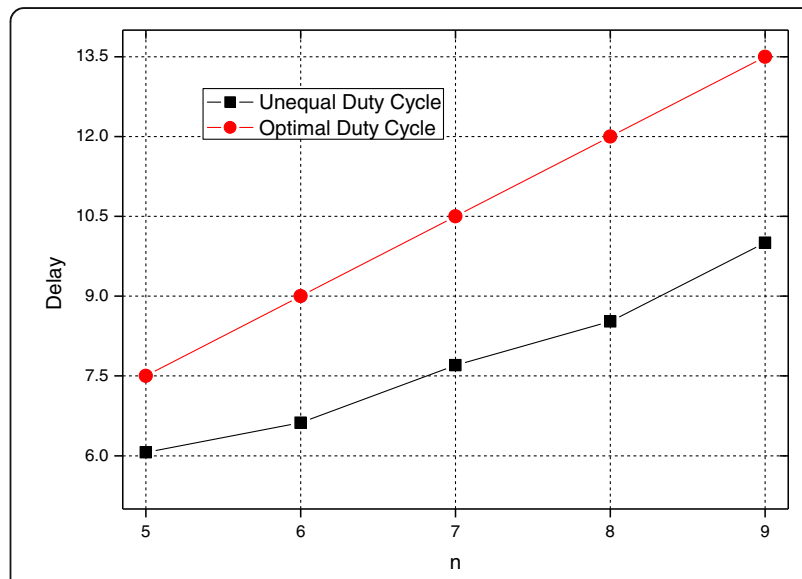

Fig. 25 The delay of UDCNP and ODCNP (MPSK) 


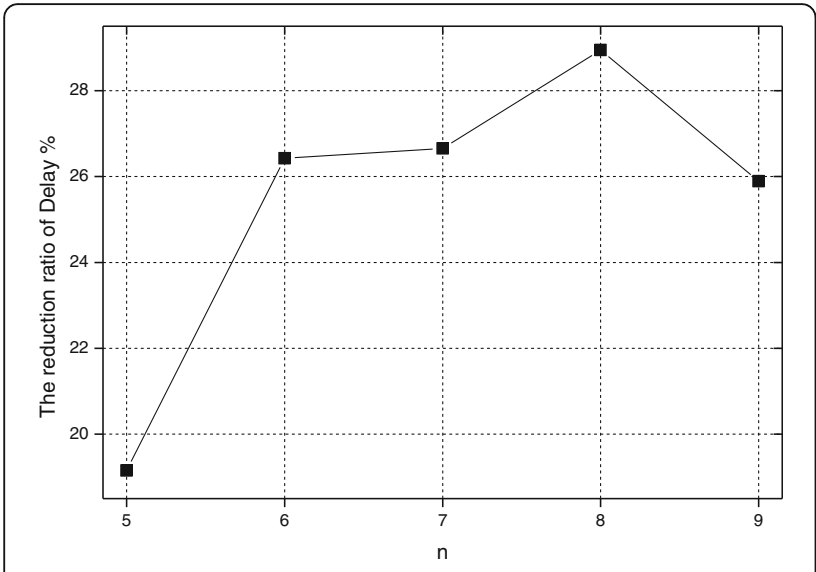

Fig. 26 The delay reduction ratio of UDCNP vs ODCNP (MPSK)

MPSK modulation technique. Theorem 21 gives the delay formula in the Grid network as follows:

Theorem 21 For Grid network under MPSK modulation technique, the delay ratio of optimization strategy in this paper to the previous strategy is given by:

$$
\phi=\frac{\sum_{i=1}^{n} \varpi\left(\tau_{1, i}\right)+\sum_{i=2}^{n} \varpi\left(\tau_{i, n}\right)}{(2 n-1) \varpi\left(\tau_{\mathrm{opt}}\right)}
$$

where the delay $\varpi(\tau)$ and $M$ are same as Theorem 10.

Proof In order to facilitate the calculation, the transmit power of each node is assumed same in the Grid network; the signal-to-noise ratio of each node will not change, so we can get:

$$
P_{e} \approx \frac{2 e^{-(\sin (\pi / M))^{2} \cdot S N R}}{b}
$$

According to (14) and (15), combined with the above formula, we can get:

$$
\varpi(\tau)=\left(1-\frac{2 B \tau T e^{-(\sin (\pi / M))^{2} \cdot S N R}}{L}\right)^{L}
$$

In the Grid network, the data is sent to the sink at most via $2 n-1$ hops, which is along the outermost edge

Table 4 The data packet forwarded of node in Grid network

\begin{tabular}{lllllll}
\hline$j$ & 1 & 2 & 3 & 4 & 5 & 6 \\
$i$ & & & & & & \\
\hline 6 & 1.969 & 1.938 & 1.875 & 1.750 & 1.500 & 1.000 \\
5 & 3.844 & 3.719 & 3.500 & 3.125 & 2.500 & 1.500 \\
4 & 5.555 & 5.266 & 4.813 & 4.125 & 3.125 & 1.750 \\
3 & 7.047 & 6.539 & 5.813 & 4.813 & 3.500 & 1.875 \\
2 & 8.293 & 7.539 & 6.539 & 5.266 & 3.719 & 1.938 \\
1 & 9.293 & 8.293 & 7.047 & 5.555 & 3.844 & 1.969 \\
\hline
\end{tabular}

Table 5 The unequal distance of nodes (MQAM)

\begin{tabular}{lllllll}
\hline$d$ & $d_{1}$ & $d_{2}$ & $d_{3}$ & $d_{4}$ & $d_{5}$ & $d_{6}$ \\
$\tau$ & & & & & & \\
\hline 0.3 & 48.43 & 71.82 & 76.69 & 84.12 & 96.49 & 122.4 \\
0.35 & 47.29 & 70.62 & 76.02 & 84.11 & 97.35 & 124.6 \\
0.4 & 45.79 & 69.08 & 75.19 & 84.15 & 98.47 & 127.3 \\
0.45 & 43.87 & 67.18 & 74.23 & 84.27 & 99.87 & 130.6 \\
0.5 & 41.46 & 64.92 & 73.19 & 84.51 & 101.6 & 134.3 \\
0.55 & 38.43 & 62.28 & 72.10 & 84.95 & 103.7 & 138.6 \\
0.6 & 34.55 & 59.28 & 71.07 & 85.64 & 106.1 & 143.3 \\
0.65 & 29.39 & 56.02 & 70.25 & 86.72 & 109.0 & 148.6 \\
0.7 & 21.80 & 52.88 & 69.97 & 88.40 & 112.5 & 154.5 \\
0.75 & 6.98 & 51.63 & 71.28 & 91.40 & 117.1 & 161.6 \\
\hline
\end{tabular}

of the network transmit to the sink. Therefore, the delay ratio is:

$$
\phi=\frac{\sum_{i=1}^{n} \varpi\left(\tau_{1, i}\right)+\sum_{i=2}^{n} \varpi\left(\tau_{i, n}\right)}{(2 n-1) \varpi\left(\tau_{\mathrm{opt}}\right)}
$$

\section{The experimental results and analysis}

This section provides some simulation examples to study the RDML scheme proposed in this paper. In the following simulation experiments, the total length of the linear network and Grid network rows (columns) are all $500 \mathrm{~m}$ and at optimal duty cycle the delay for each node is taken as 1.5. We define the unequal distance of nodes policy as UDNP, the equal distance of nodes policy as EDNP [49], the unequal duty cycle of nodes policy as UDCNP, and the optimal duty cycle of nodes policy as ODCNP [55].

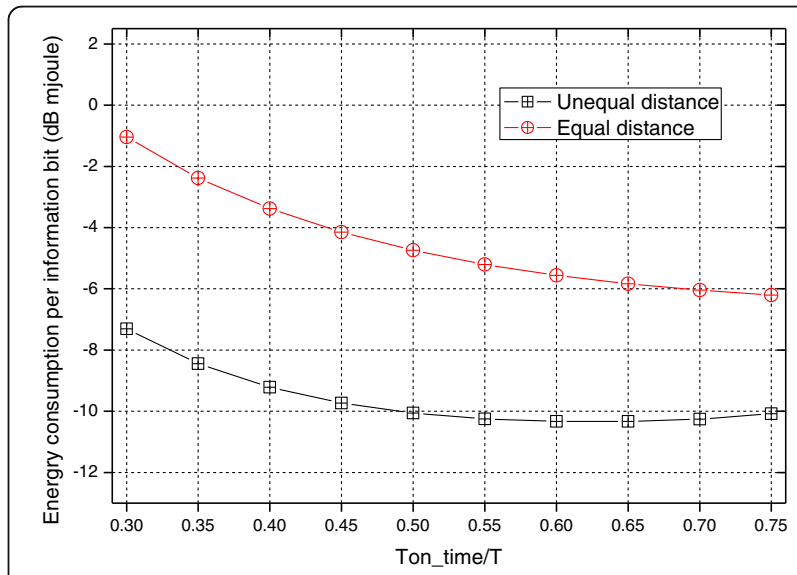

Fig. 27 The maximum energy consumption under UDNP and EDNP (MQAM) 


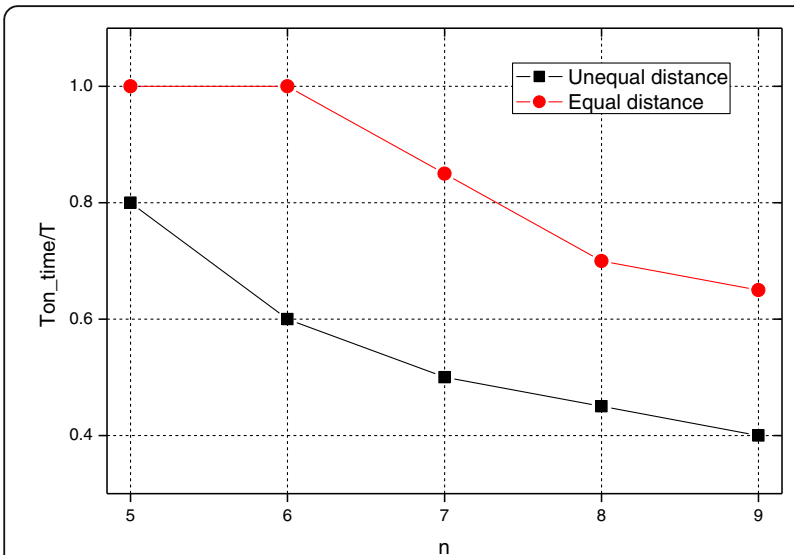

Fig. 28 The optimal duty cycle under UDNP and EDNP (MQAM)

\subsection{Linear network}

\subsubsection{Performance in MQAM technique}

For EDNP, the energy consumption of nodes is unbalanced due to the different data packets of each node. Therefore, UDNP strategy is proposed. The following will verify our strategy by some simulation examples.

For UDNP, according to Eq. (18), we can get the distance between nodes in a $n=6$ linear network as shown in Table 2.

Figure 9 shows the maximum energy consumption under UDNP and EDNP, where the horizontal axis represents the duty cycle and the vertical axis represents the maximum energy consumption for transmitting a bit data in terms of decibels relative to a $10^{-3} \mathrm{~J}: 10 \log _{10}\left(E_{\text {infBit }}\right.$. $\left.10^{3}\right) \mathrm{dB} \mathrm{mJ}$. It can be seen for UDNP that the minimum of maximum energy consumption achieved at a duty cycle is 0.55, compared with EDNP [49] in which the maximum energy consumption decreased by $2.80667 \mathrm{~dB}$.

Next, we will consider the maximum energy consumption of UDNP and EDNP in networks with different

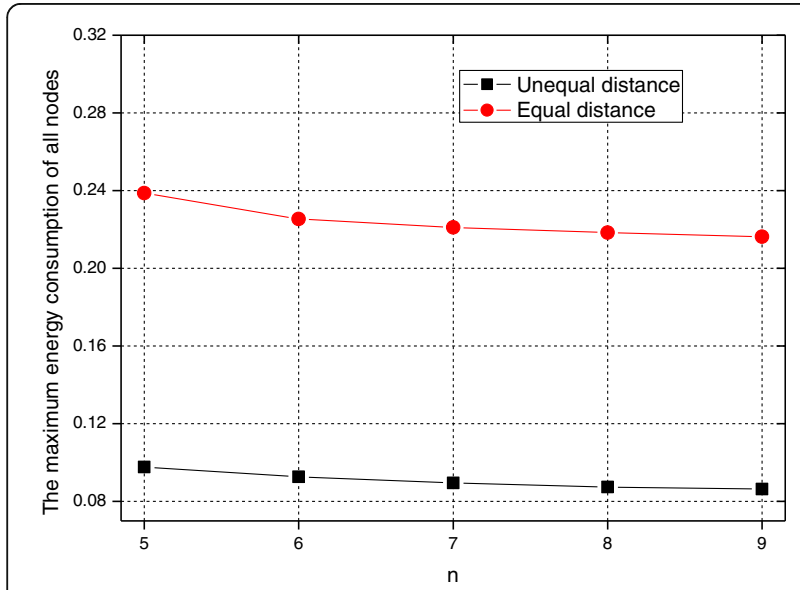

Fig. 29 The maximum energy consumption at optimal duty cycle under UDNP and EDNP (MQAM)

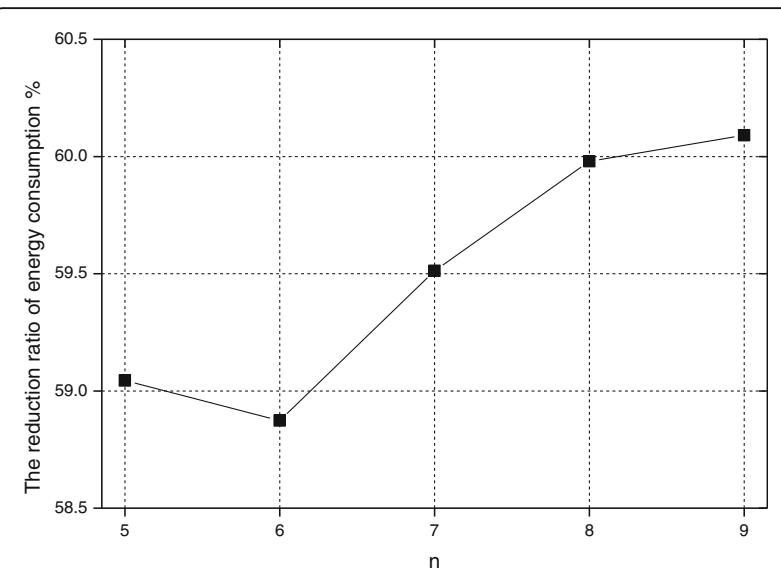

Fig. 30 The maximum energy consumption reduction ratio of UDNP vs EDNP (MQAM)

number of nodes when both strategies adopt an optimal duty cycle.

Figure 10 shows the optimal duty cycle of UDNP and EDNP in the linear network with $n$ nodes, where the horizontal axis represents the number of nodes in the network and the vertical axis represents the optimal duty cycle. Figure 11 shows the maximum energy consumption of all nodes in the network when both strategies are at the optimal duty cycle. As shown in Fig. 12, it can be seen the UDNP scheme is reducing the maximum energy consumption by more than $43 \%$ over the EDNP scheme [49].

We used to deploy the nodes into a non-equidistant method to optimize the network lifetime. In addition, when the nodes are deployed equidistantly, we can use a strategy of unequal duty cycle, near the sink nodes using the optimal duty cycle to ensure the network lifetime and away from the sink node appropriate to increase the duty cycle to reduce the delay.

Figure 13 shows the duty cycle used by each node at a $n=6$ linear network under UDCNP and ODCNP, where the horizontal axis is the node number and the vertical is the selected duty cycle. Figure 14 shows the delay of each node under UDNCP and ODCNP, where the horizontal axis presents the node number and the vertical

Table 6 The duty cycle used at each node (MQAM)

\begin{tabular}{lllllll}
\hline$j$ & 1 & 2 & 3 & 4 & 5 & 6 \\
$i$ & & & & & & \\
\hline 6 & 1 & 1 & 1 & 1 & 1 & 1 \\
5 & 1 & 1 & 1 & 1 & 1 & 1 \\
4 & 1 & 1 & 1 & 1 & 1 & 1 \\
3 & 1 & 1 & 1 & 1 & 1 & 1 \\
2 & 0.8 & 0.9 & 1 & 1 & 1 & 1 \\
1 & 0.7 & 0.8 & 1 & 1 & 1 & 1 \\
\hline
\end{tabular}




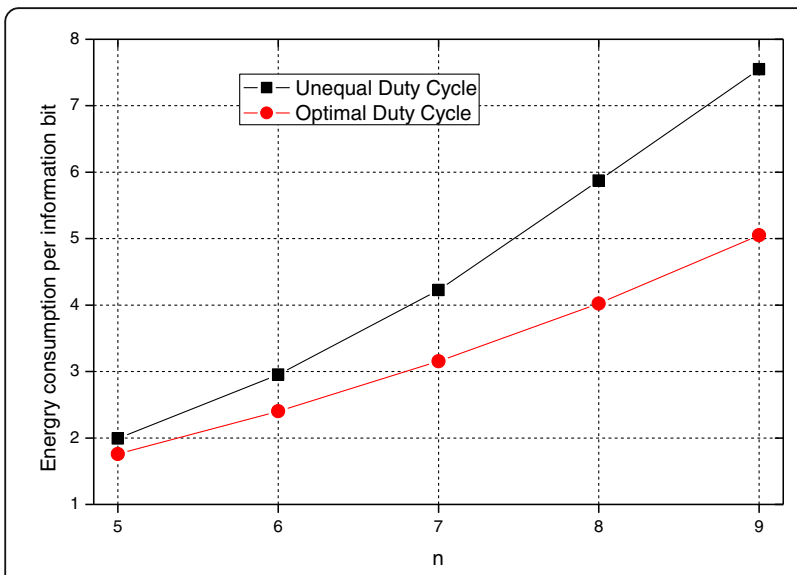

Fig. 31 The total energy consumption in Grid network under UDCNP and OPCNP (MQAM)

presents the delay from each node to sink. It can be seen that the UDCNP scheme is reducing the maximum delay by 2.48116 over the ODCNP scheme [55].

Figure 15 shows the total energy consumption of the network with $n$ nodes under UDCNP and ODCNP, where the horizontal presents the number of nodes in the network and the vertical presents the total energy consumption of the network. It can be seen the energy consumption of UDCNP is higher than that of ODCNP, but we reduce the network delay by increasing the duty cycle of the node that is away from the sink. Figure 16 is the network delay under UDCNP and ODCNP, combined with Fig. 17 which shows UDCNP is reducing the network delay by more than $25 \%$ over ODCNP.

\subsubsection{Performance in MPSK technique}

For MPSK modulation technique, we can also use UDNP to optimize the network lifetime.

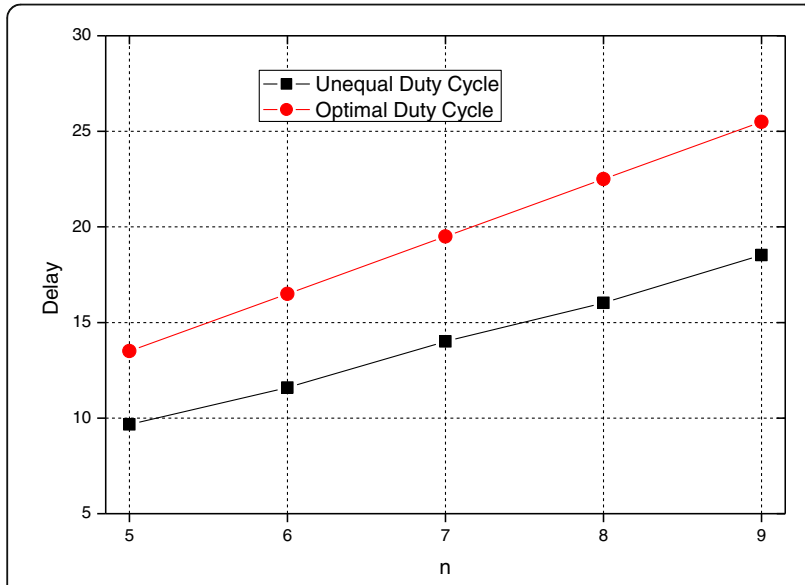

Fig. 32 The delay in Grid network under UDCNP and ODCNP (MQAM)

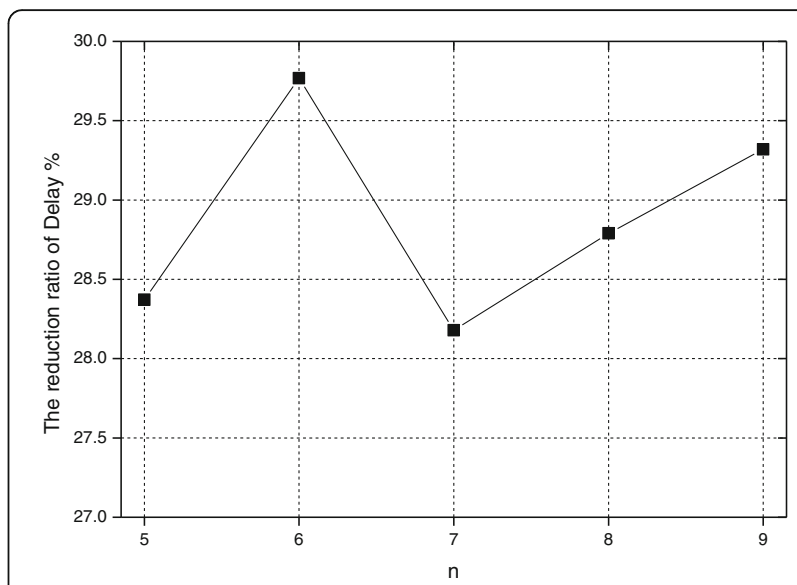

Fig. 33 The delay reduction of UDCNP vs ODCNP (MQAM)

According to Eq. (22), we can calculate the distance between the nodes as in Table 3 .

Figure 18 is the maximum energy consumption at linear network with $n=6$. It can be seen that when UDNP uses the optimal duty cycle, the maximum energy consumption is reduced by $2.80127 \mathrm{~dB}$ over that of EDNP.

Figure 19 is the optimal duty cycle of the network at the MPSK modulation technique. Figure 20 is the maximum energy consumption at the optimal duty cycle of UDNP and EDNP. From Fig. 21, it can be seen the UDNP scheme is reducing the maximum energy consumption by more than $44 \%$ over the EDNP scheme.

Figure 22 shows the duty cycle used by each node at a $n=6$ linear network under the MPSK modulation technique. Figure 23 is the delay of each node in the network under the MPSK modulation technique. Similarly, the maximum delay for the UDCNP scheme is reduced by 2.37812 compared to that for the ODCNP scheme.

Table 7 The unequal distance of nodes (MPSK)

\begin{tabular}{lllllll}
\hline$d$ & 1 & 2 & 3 & 4 & 5 & 6 \\
$\tau$ & & & & & & \\
\hline 0.35 & 48.00 & 71.36 & 76.43 & 84.11 & 96.81 & 123.26 \\
0.4 & 46.34 & 69.64 & 75.49 & 84.13 & 98.06 & 126.35 \\
0.45 & 44.15 & 67.46 & 74.37 & 84.25 & 99.67 & 130.12 \\
0.5 & 41.46 & 64.92 & 73.19 & 84.51 & 101.59 & 134.33 \\
0.55 & 38.29 & 62.17 & 72.06 & 84.97 & 103.75 & 138.77 \\
0.6 & 34.63 & 59.34 & 71.09 & 85.63 & 106.05 & 143.24 \\
0.65 & 30.42 & 56.60 & 70.37 & 86.50 & 108.45 & 147.63 \\
0.7 & 25.49 & 54.16 & 69.99 & 87.59 & 110.89 & 151.86 \\
0.75 & 19.41 & 52.29 & 70.06 & 88.92 & 113.39 & 155.94 \\
0.8 & 11.10 & 51.49 & 70.77 & 90.61 & 116.05 & 159.99 \\
\hline
\end{tabular}




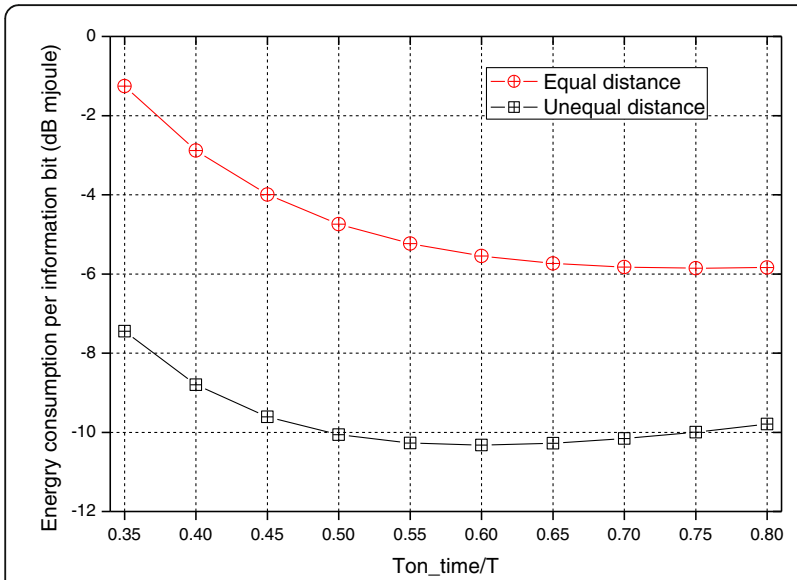

Fig. 34 The maximum energy consumption under UDNP and EDNP (MPSK)

Figure 24 is the total energy consumption of the network using the MPSK modulation technique, and it can be seen the total energy consumption of UDCNP is higher than that of ODCNP. Figure 25 is the delay of network under the MPSK modulation technique. Combined with Fig. 26, it can be seen the delay for the UDCNP scheme is reduced by more than $19 \%$ over that for the ODCNP scheme.

\subsection{Grid network}

This section verifies the optimization strategy of the Grid network. We first choose a $6 \times 6$ Grid network model. According to Eq. (24), the data packets forwarded by each node can be calculated in Grid network as in Table 4.

\subsubsection{Performance in MQAM technique}

For a $6 \times 6$ Grid network under the MQAM modulation technique, when adopting UDNP, according to Eq. (29) and Table 4, we can calculate the distance as in Table 5.

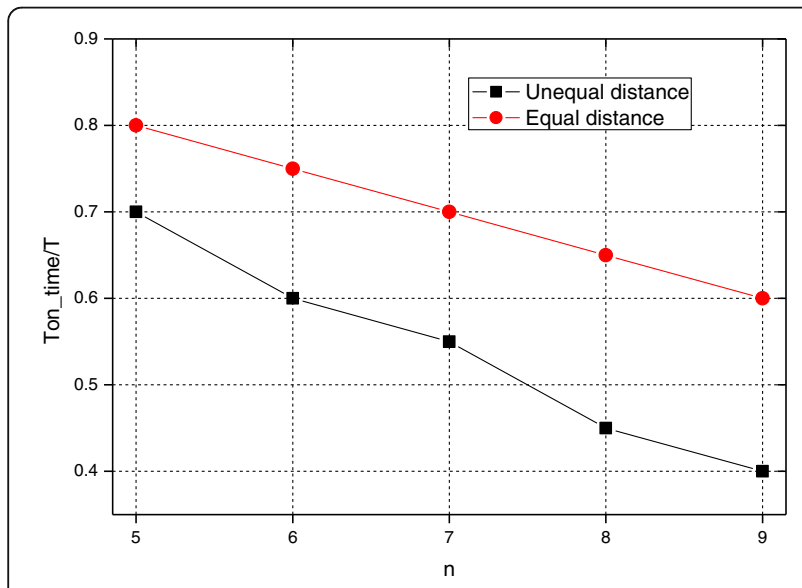

Fig. 35 The optimal duty cycle under UDNP and EDNP (MPSK)

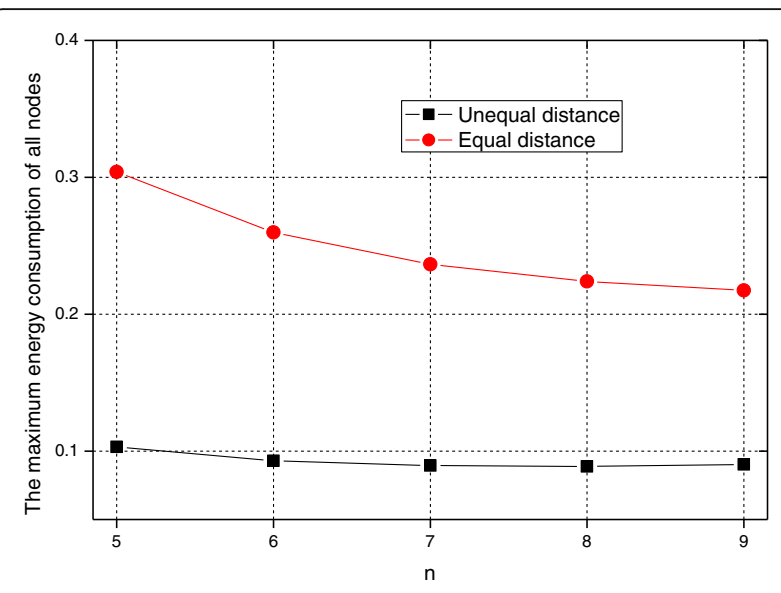

Fig. 36 The maximum energy consumption at optimal duty cycle under UDNP and EDNP (MPSK)

Figure 27 shows the maximum energy consumption of a node in the Grid network when the energy consumption of the outermost nodes is the same. In the Grid network, the amount of data packets increases, so the gap between the two strategies will also increase. When UDNP uses the optimal duty cycle, the maximum energy consumption is less than half of that of EDNP.

Figure 28 is the optimal duty cycle under UDNP and EDNP. In EDNP, when the number of nodes is small, due to the distance of $d_{1}$ which is longer compared with that of linear network, the minimum energy consumption can be obtained when the duty cycle is maximum. Figure 29 is the maximum energy consumption under UDNP and EDNP. From Fig. 30, it can be seen the maximum energy consumption of UDNP is reduced by more than $58 \%$ over that of EDNP [49].

In a Grid network with $n=6$, the duty cycle of each node under the MQAM modulation technique is as Table 6 when the lifetime of the Grid network cannot be reduced.

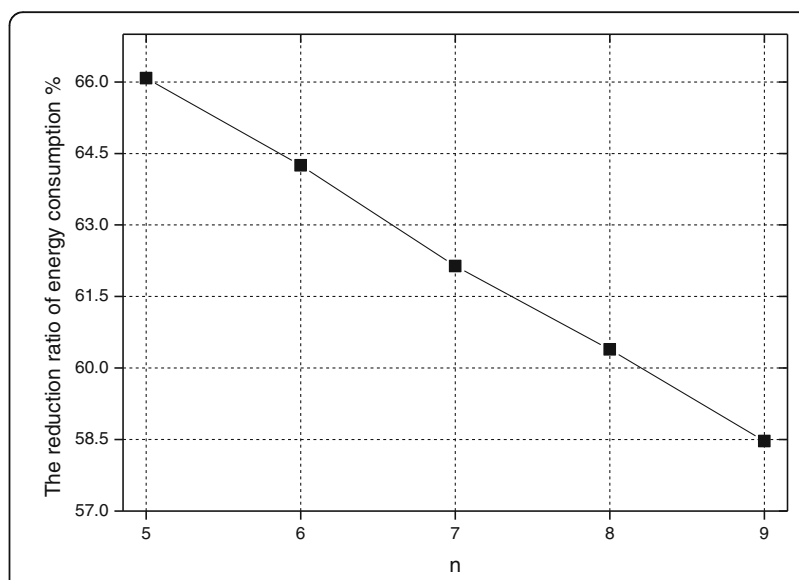

Fig. $\mathbf{3 7}$ The maximum energy consumption reduction ratio of UDNP vs EDNP (MPSK) 
Table 8 The duty cycle used at each node (MPSK)

\begin{tabular}{lllllll}
\hline$j$ & 1 & 2 & 3 & 4 & 5 & 6 \\
$i$ & & & & & & \\
\hline 6 & 1 & 1 & 1 & 1 & 1 & 1 \\
5 & 1 & 1 & 1 & 1 & 1 & 1 \\
4 & 1 & 1 & 1 & 1 & 1 & 1 \\
3 & 0.9 & 1 & 1 & 1 & 1 & 1 \\
2 & 0.7 & 0.8 & 1 & 1 & 1 & 1 \\
1 & 0.6 & 0.7 & 0.9 & 1 & 1 & 1 \\
\hline
\end{tabular}

Figure 31 is the total energy consumption of the Grid network under UDCNP and ODCNP. The total energy consumption of UDCNP is higher than that of ODCNP. Figure 32 shows the delay of UDCNP and ODCNP. From Fig. 33, it can be seen that the delay of UDCNP is reduced by more than $28 \%$ over that of ODCNP [55].

\subsubsection{Performance in MPSK technique}

When the modulation technique is MPSK, choose the same Grid network as in Section 5.2.1. According to Eq. (33), we can get the distance between the nodes as in Table 7 .

Figure 34 is the maximum energy consumption of a node in Grid network. It can be seen the optimal duty cycle is 0.6 and the maximum energy consumption of UDNP is less than half of that of EDNP.

Figure 35 is the maximum energy consumption of Grid network when the energy consumption of the outermost nodes is the same. Figure 36 is the optimal duty cycle under the MPSK modulation technique. From Fig. 37, it can be seen that the maximum energy consumption of UDNP is reduced by more than $58 \%$ over that of EDNP.

In a Grid network with $n=6$, the duty cycle of each node under MPSK modulation technique is as in Table 8 when the lifetime of the Grid network cannot be reduced.

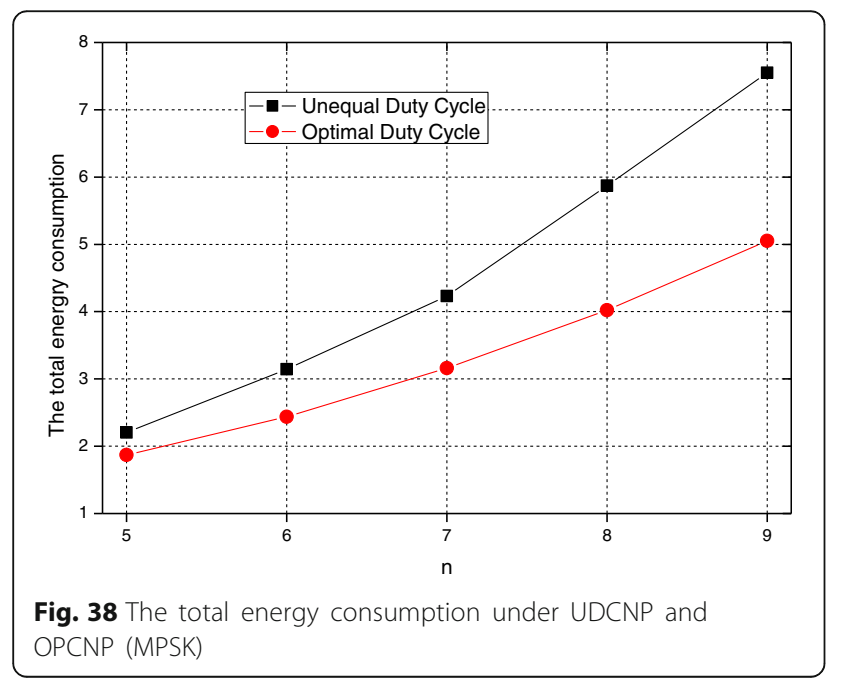

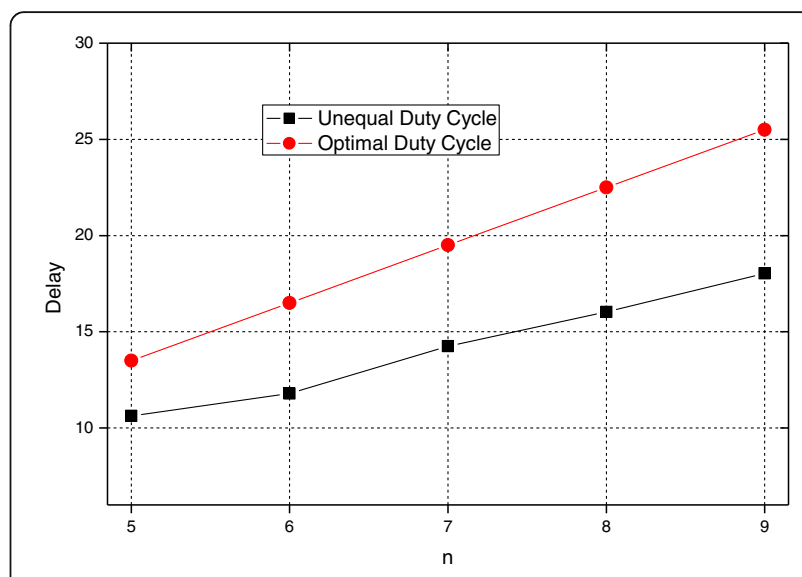

Fig. 39 The delay of UDCNP and ODCNP (MPSK)

Figure 38 is the total energy consumption of UDCNP and ODCNP, and the total energy consumption of UDCNP is higher than that of ODCNP. Figure 39 shows the delay of UDNCP and ODCNP, and combined with Fig. 40, we can see that the delay of UDCNP is less than that of ODCNP by more than $22 \%$.

\section{Conclusions}

In this paper, we propose a cross-layer design scheme for IWSNs which is from duty cycle and node deployment and other layers to optimize the network lifetime and delay. This paper discusses the linear network and Grid network that use two modulation techniques which are the MQAM technique and the MPSK technique to optimize the performance of network under additive white Gaussian noise channels.

Different from the previous research, the previous optimization strategies choose to increase the energy efficiency of the node and optimize the network lifetime under a certain duty cycle. The strategy of this paper is to further

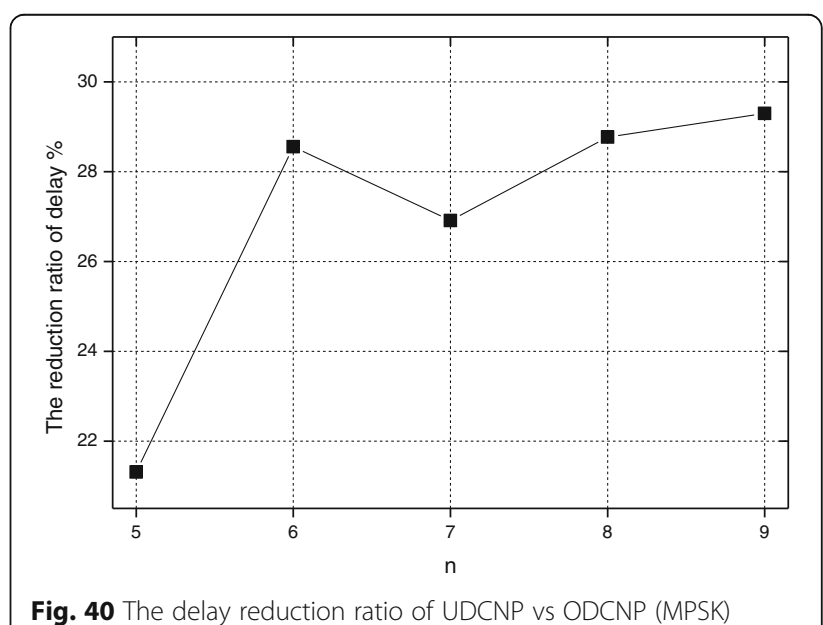


optimize the network lifetime by adjusting the duty cycle when the energy efficiency has reached an optimal value.

However, in reality, the node deployment difficulty and deviation are considered, and the energy consumption of the node cannot be completely balanced, so we put forward a strategy of unequal duty cycle. For energyintensive nodes, the duty cycle uses the optimal value to optimize the network lifetime; for those nodes with low energy consumption, the duty cycle is appropriately increased to reduce the transmission delay. This strategy can be applied to industrial wireless sensor networks, can increase the lifetime of IWSNs, and can speed up the response of the network to events. Therefore, this strategy has a good meaning.

\section{Acknowledgements}

This work was supported in part by the National Natural Science Foundation of China $(61772554,61379110,61572526,61572528)$ and the National Basic Research Program of China (973 Program) (2014CB046305).

\section{Funding}

Not applicable.

\section{Availability of data and materials}

Not applicable.

\section{Authors' contributions}

$J T$ is the main author of the current paper. AL contributed to the conception and design of the study. MZ, HS, and MM commented the work. All authors read and approved the final manuscript.

\section{Competing interests}

The authors declare that they have no competing interests.

\section{Publisher's Note}

Springer Nature remains neutral with regard to jurisdictional claims in published maps and institutional affiliations.

\section{Author details}

'School of Information Science and Engineering, Central South University, Changsha 410083, China. ${ }^{2}$ School of Software, Central South University, Changsha 410075, China. ${ }^{3}$ Department of Computer Science, Stony Brook University, New York, NY 11794, USA.

Received: 27 December 2017 Accepted: 9 February 2018

Published online: 01 March 2018

\section{References}

1. H Zheng, W Guo, N Xiong, A kernel-based compressive sensing approach for mobile data gathering in wireless sensor network systems. IEEE Transactions on Systems. Man Cybern. Syst. (2017). https://doi.org/10.1109/ TSMC.2017.2734886.

2. J Gao, J Wang, P Zhong, $\mathrm{H}$ Wang, On threshold free error detection for industrial wireless sensor networks. IEEE Trans. Ind. Inform. (2017). https:// doi.org/10.1109/TII.2017.2785395.

3. J Gao, Q Ping, J Wang, Resisting re-identification mining on social graph data. World Wide Web. (2018). https://doi.org/10.1007/s11280-017-0524-3

4. A Shahzad, M Lee, HD Kim, S Woo, N Xiong, New security development and trends to secure the SCADA sensors automated transmission during critical sessions. Symmetry 7(4), 1945-1980 (2015). https://doi.org/10.3390/ sym7041945.

5. N Xiong, RW Liu, M Liang, D Wu, Z Liu, H Wu, Effective alternating direction optimization methods for sparsity-constrained blind image deblurring. Sensors 17(1), 174 (2017). https://doi.org/10.3390/s17010174.
6. W Cheng, M Zhao, N Xiong, KT Chui, Non-convex sparse and low-rank based robust subspace segmentation for data mining. Sensors (2017). https://doi.org/10.3390/s17071633.

7. Z Ning, X Wang, X Kong, W Hou, A social-aware group formation framework for information diffusion in narrowband Internet of things. IEEE Internet Things J. (2017). https://doi.org/10.1109/JIOT.2017.2777480.

8. Y Xu, A Liu, C Huang, Delay-aware program codes dissemination scheme in Internet of everything. Mob. Inf. Syst. (2016). https://doi.org/10.1155/2016/2436074.

9. X Liu, S Zhao, A Liu, N Xiong, AV Vasilakos, Knowledge-aware proactive nodes selection approach for energy management in Internet of things. Futur. Gener. Comput. Syst. (2017). https:/doi.org/10.1016/j.future.2017.07.022.

10. Q Zhang, A Liu, An unequal redundancy level-based mechanism for reliable data collection in wireless sensor networks. EURASIP J. Wirel. Commun. Netw. 2016(1), 258 (2016)

11. A Liu, X Liu, Z Tang, LT Yang, Z Shao, Preserving smart sink location privacy with delay guaranteed routing scheme for WSNs. ACM Trans. Embed. Comput. Syst. 16(3), 68 (2017)

12. X Liu, A Liu, Z Li, S Tian, YJ Choi, H Sekiya, J Li, Distributed cooperative communication nodes control and optimization reliability for resourceconstrained WSNs. Neurocomputing 270, 122-136 (2017)

13. X Chen, M Ma, A Liu, Dynamic power management and adaptive packet size selection for loT in e-healthcare. Comput. Electrical Eng. (2017). https:// doi.org/10.1016/j.compeleceng.2017.06.010.

14. X Liu, G Li, S Zhang, A Liu, Big program code dissemination scheme for emergency software-define wireless sensor networks. Peer-to-Peer Netw. Appl. 2017 (2017). https://doi.org/10.1007/s12083-017-0565-5.

15. RW Liu, L Shi, SCH Yu, N Xiong, D Wang, Reconstruction of undersampled big dynamic MRI data using non-convex low-rank and sparsity constraints. Sensors 17(3), 509 (2017)

16. J Xu, X Liu, M Ma, A Liu, T Wang, C Huang, Intelligent aggregation based on content routing scheme for cloud computing. Symmetry 9(10), 221 (2017)

17. Z Ning, X Hu, Z Chen, M Zhou, B Hu, J Cheng, M Obaidat, A cooperative quality aware service access system for social Internet of vehicles. IEEE Int. Things J. (2017). https://doi.org/10.1109/JIOT.2017.2764259.

18. A Liu, Z Chen, N Xiong, An adaptive virtual relaying set scheme for lossand-delay sensitive WSNs. Inf. Sci. 424, 118-136 (2018)

19. M Huang, A Liu, T Wang, C Huang, Green data gathering under delay differentiated services constraint for Internet of things. Wirel. Commun. Mob. Comput. 2018, 9715428 (2018). https://doi.org/10.1155/2018/9715428.

20. T Li, Y Liu, L Gao, A Liu, A cooperative-based model for smart-sensing tasks in fog computing. IEEE Access 5, 21296-21311 (2017)

21. Z Wang, Q Cao, H Qi, H Chen, Q Wang, Cost-effective barrier coverage formation in heterogeneous wireless sensor networks. Ad Hoc Netw. 64(9), 65-79 (2017)

22. Y Liu, A Liu, S Guo, Z Li, YJ Choi, H Sekiya, Context-aware collect data with energy efficient in cyber-physical cloud systems. Futur. Gener. Comput. Syst. (2017). https://doi.org/10.1016/j.future.2017.05.029.

23. Y Xu, X Chen, A Liu, C Hu, A latency and coverage optimized data collection scheme for smart cities based on vehicular ad-hoc networks. Sensors 17, 888 (2017)

24. K Xie, J Cao, X Wang, J Wen, Optimal resource allocation for reliable and energy efficient cooperative communications. IEEE Trans. Wirel. Commun. 12(10), 4994-5007 (2013)

25. J Xu, A Liu, N Xiong, T Wang, Z Zuo, Integrated collaborative filtering recommendation in social cyber-physical systems. Int. J. Distributed Sens. Netw. 13(12), 1550147717749745 (2017)

26. F Ma, X Liu, A Liu, M Zhao, C Huang, T Wang, A time and location correlation incentive scheme for deeply data gathering in crowdsourcing networks. Wirel. Commun. Mob. Comput. 2018, 8052620 (2018). https://doi. org/10.1155/2018/8052620.

27. X Liu, Y Liu, H Song, A Liu, Big data orchestration as a service networking. IEEE Commun. Mag. 55(9), 94-101 (2017)

28. J Wang, A Liu, S Zhang, Key parameters decision for cloud computing: insights from a multiple game model. Concurrency Comput. Pract. Exp. (2017). https://doi.org/10.1002/cpe.4200.

29. M Wu, Y Wu, X Liu, M Ma, A Liu, M Zhao, Learning based synchronous approach from forwarding nodes to reduce the delay for industrial Internet of things. EURASIP J. Wirel. Commun. Netw. 2018, 10 (2018). https://doi.org/ 10.1186/s13638-017-1015-z.

30. K Ota, M Dong, J Gui, A Liu, QUOIN: incentive mechanisms for crowd sensing networks. IEEE Netw. Mag. (2017). https://doi.org/10.1109/MNET.2017.1500151. 
31. S Manfredi, E Di Tucci, Decentralized control algorithm for fast monitoring and efficient energy consumption in energy harvesting wireless sensor networks. IEEE Trans. Ind. Inform. 13(4), 1513-1520 (2017)

32. M Farooq-I-Azam, Q Ni, EA Ansari, Intelligent energy efficient localization using variable range beacons in industrial wireless sensor networks. IEEE Trans. Ind. Inform. 12(6), 2206-2216 (2016)

33. Z Wang, J Liao, Q Cao, H Qi, Z Wang, Achieving k-barrier coverage in hybrid directional sensor networks. IEEE Trans. Mob. Comput. 13(7), 1443-1455 (2014)

34. KXie, X Wang, J Wen, J Cao, Cooperative routing with relay assignment in multi-radio multi-hop wireless networks. IEEE/ACM Trans. Networking 24(2), 859-872 (2016)

35. Q Liu, A Liu, On the hybrid using of unicast-broadcast in wireless sensor networks. Comput. Electrical Eng. (2017). https://doi.org/10.1016/j. compeleceng.2017.03.004

36. X Chen, Y Xu, A Liu, Cross layer design for optimal delay, energy efficiency and lifetime in body sensor networks. Sensors 17(4), 900 (2017). https://doi. org/10.3390/s17040900.

37. H Cheng, Z Su, N Xiong, Y Xiao, Energy-efficient nodes scheduling algorithms for wireless sensor networks using Markov random field model. Inf. Sci. 329, 461-477 (2016)

38. X Liu, M Dong, K Ota, LT Yang, A Liu, Trace malicious source to guarantee cyber security for mass monitor critical infrastructure. J. Comput. Syst. Sci. (2016). https://doi.org/10.1016/j.jcss.2016.09.008

39. J Tang, A Liu, M Zhao, T Wang, An aggregate signature based trust routing for data gathering in sensor networks. Secur. Commun. Netw. (2018). https://doi.org/10.1155/2018/6328504

40. K Huang, Q Zhang, C Zhou, N Xiong, Y Qin, An efficient intrusion detection approach for visual sensor networks based on traffic pattern learning. IEEE Trans. Syst. Man. Cybern. 1(99), 1-10 (2017). https://doi.org/10.1109/TSMC. 2017.2698457

41. Q Fan, N Xiong, K Zeitouni, Q Wu, A Vasilakos, YC Tian, Game balanced multi-factor multicast routing in sensor grid networks. Inf. Sci. 367, 550-572 (2016). https://doi.org/10.1016/j.ins.2016.06.049

42. J Gui, K Zhou, Flexible adjustments between energy and capacity for topology control in heterogeneous wireless multi-hop networks. J. Netw. Syst. Manag. 24, 789-812 (2016)

43. V Agarwal, RA DeCarlo, LH Tsoukalas, Modeling energy consumption and lifetime of a wireless sensor node operating on a contention-based MAC protocol. IEEE Sensors J. 17(16), 5153-5168 (2017)

44. M Zhou, M Zhao, A Liu, M Ma, T Wang, C Huang, Fast and efficient data forwarding scheme for tracking mobile target in sensor networks. Symmetry. 9(11), 269 (2017)

45. H Cheng, Y Chen, N Xiong, F Li, Layer-based data aggregation and performance analysis in wireless sensor networks. J. Appl. Math. 2013, (2016). https://doi.org/10.1155/2013/502381

46. A Liu, S Zhao, High performance target tracking scheme with low prediction precision requirement in WSNs. Int. J. Ad Hoc. Ubiquitous Comput. (2016). http://www.inderscience.com/info/ingeneral/forthcoming. php?jcode=ijahuc

47. J Gao, J Wang, J He, F Yan, Against signed graph deanonymization attacks on social network. Int. J. Parallel Prog. (2017). https://doi.org/10.1007/ s10766-017-0546-6.

48. N Xiong, AV Vasilakos, LT Yang, L Song, Y Pan, R Kannan, Y Li, Comparative analysis of quality of service and memory usage for adaptive failure detectors in healthcare systems. IEEE J. Selected Areas Commun. 27(4), 495-509 (2009)

49. X Chen, Y Hu, A Liu, Z Chen, Cross layer optimal design with guaranteed reliability under Rayleigh block fading channels. KSII Trans. Internet Inf. Syst. 7(12), 3071-3095 (2013)

50. J Long, A He, A Liu, X Chen, Adaptive sensing with reliable guarantee under white Gaussian noise channels of sensor networks. J. Sensors 2015, 532045 (2015). https://doi.org/10.1155/2015/532045.

51. J Long, M Dong, K Ota, A Liu, S Hai, Reliability guaranteed efficient data gathering in wireless sensor networks. IEEE Access 3, 430-444 (2015)

52. S Ezdiani, IS Acharyya, S Sivakumar, A Al-Anbuky, Wireless sensor network softwarization: towards WSN adaptive QoS. IEEE Int. Things J. 4(5), 1517-1527 (2017)

53. R Anane, K Raoof, R Bouallegue, Minimization of wireless sensor network energy consumption through optimal modulation scheme and channel coding strategy. J. Signal Process. Syst. 83(1), 65-81 (2016)
54. A Boukerche, X Zhou, MAC transmission protocols for delay-tolerant sensor networks. Comput. Netw. 124, 108-125 (2017)

55. R Zhang, O Berder, J Gorce, O Sentieys, Energy-delay tradeoff in wireless multihop networks with unreliable links. Ad Hoc Netw. 10, 1306-1321 (2012)

56. P Chen, B O'Dea, E Callaway, Energy efficient system design with optimum transmission range for wireless ad hoc networks. Proc. IEEE Int. Conf. Commun. 2, 945-952 (2002)

57. $J$ Gao, Analysis of energy consumption for ad hoc wireless sensor networks using a bit-meter-per-joule metric, Jet Propulsion Laboratory, California Institute of Technology. Tech. Rep. 42,150 (2002)

58. Y Chen, C Chuah, Q Zhao, Network configuration for optimal utilization efficiency of wireless sensor networks. Ad Hoc Netw. 6, 92-107 (2008)

59. PF Wu, F Xiao, C Sha, HP Huang, RC Wang, N Xiong, Node scheduling strategies for achieving full-view area coverage in camera sensor networks. Sensors 17(6), 1303 (2017). https://doi.org/10.3390/s17061303.

60. M Tahir, R Farrell, A cross-layer framework for optimal delay-margin, network lifetime and utility tradeoff in wireless visual sensor networks. Ad Hoc Netw. 11, 701-711 (2013)

61. A Liu, Z Zheng, C Zhang, Z Chen, X Shen, Secure and energy-efficient disjoint multi-path routing for WSNs. IEEE Trans. Veh. Technol. 61(7), 3255-3265 (2012)

62. Q Fan, K Zeitouni, N Xiong, Q Wu, A General Nash Equilibrium Semantic Cache Algorithm in a sensor grid database system. IEEE Trans. Syst. Man. Cybern. 4(99) (2016). https://doi.org/10.1109/TSMC.2016.2523949.

\section{Submit your manuscript to a SpringerOpen ${ }^{\circ}$ journal and benefit from:}

- Convenient online submission

- Rigorous peer review

- Open access: articles freely available online

- High visibility within the field

- Retaining the copyright to your article

Submit your next manuscript at $>$ springeropen.com 